\title{
Metric Temporal Logic with Counting
}

\author{
Khushraj Madnani ${ }^{1}$, Shankara Narayanan Krishna ${ }^{1}$ and Paritosh K. Pandya ${ }^{2}$ \\ 1 Department of Computer Science \& Engineering, IIT Bombay, Mumbai, India 400076. \\ \{khushraj,krishnas\}@cse.iitb.ac.in \\ 2 School of Technology and Computer Science, Tata Institute of Fundamental Research, \\ Mumbai, India 400005 \\ pandya@tcs.tifr.res.in
}

\begin{abstract}
Ability to count number of occurrences of events within a specified time interval is very useful in specification of resource bounded real time computation. In this paper, we study an extension of Metric Temporal Logic (MTL) with two different counting modalities called C and UT (until with threshold), which enhance the expressive power of MTL in orthogonal fashion. We confine ourselves only to the future fragment of MTL interpreted in a pointwise manner over finite timed words. We provide a comprehensive study of the expressive power of logic CTMTL and its fragments using the technique of EF games extended with suitable counting moves. Finally, as our main result, we establish the decidability of CTMTL by giving an equisatisfiable reduction from CTMTL to MTL. The reduction provides one more example of the use of temporal projections with oversampling introduced earlier for proving decidability. Our reduction also implies that MITL extended with C and UT modalities is elementarily decidable.
\end{abstract}

\section{Introduction}

Temporal logics provide constructs to specify qualitative ordering between events in time. But real time logics have the ability to specify quantitative timing constraints between events. Metric Temporal Logic MTL is amongst the best studied of real time logics. Its principle modality $a \mathrm{U}_{I} b$ states that an event $b$ should occur in future within a time distance lying within interval $I$. Moreover, $a$ should hold continuously till then.

In many situations, especially those dealing with resource bounded computation, the ability to count the number of occurrences of events becomes important. In this paper, we consider an extension of MTL with two counting modalities C and UT (until threshold) which provide differing abilities to specify constraints on counts on events in time intervals. The resulting logic is called CTMTL. Modality $C_{I}^{\geq n} \phi$ states that the number of times formula $\phi$ holds in time interval $I$ (measured relative to current time point) is at least $n$. This is a mild generalization of $\mathrm{C}_{(0,1)}^{\geq n} \phi$ modality studied by Rabinovich [1] in context of continuous time MTL. The UT modality $\phi \mathrm{U}_{I, \# \kappa \geq n} \psi$ is like MTL until but it additionally states that the number of time formula $\kappa$ holds between now and time point where $\psi$ holds is at least $n$. Thus it extends $\mathrm{U}$ to simultaneously specify constraint on time and count of subformula. Constraining $U$ by count of subformula was already explored for untimed LTL by Laroussini et al [5]. But the combination of timing and counting seems new. The following example illustrates the use of these modalities. An Example. We specify some constraints to be monitored by exercise bicycle electronics. 
- Two minutes after the start of exercise, the heartbeat (number of pulses in next 60 seconds) should be between 90 and 120 . This can be stated as $\square\left(\right.$ st $\Rightarrow\left(\mathrm{C}_{[120,180]}^{>90}\right.$ pulse $\wedge \mathrm{C}_{[120,180]}^{<120}$ pulse $\left.)\right)$

- Here is one exerise routine: After start of exercise, slow_peddling should be done for 1 kilometre (marked by odometer giving 1000 pulses) and this should be achieved in interval 1 to 2 minutes. After this fast_peddling should be done for 3 minutes. This can be specified as $\square\left(\right.$ st $\Rightarrow$ slowpeddle $\mathrm{U}_{[60,120] \text {,\#odo=1000 }}\left(\square_{[0,180]}\right.$ fastpeddle $\left.)\right)$

The expressiveness and decidability properties of real time logics differ considerable based on nature of time. There has been considerable study of counting MTL in continuous time [11],[2]. In this paper, we consider the case of pointwise time, i.e. CTMTL interpreted over finite timed words in a pointwise manner. We provide a comprehensive picture of expressiveness and decidability of CTMTL and its fragments in pointwise time and we find that this differs considerably when compared with continuous time.

As our first main result, we show that the $C$ and the UT modalities both increase the expressive power of MTL but they are mutually incomparable. EF games are a classical technique used to study expressive power of logic. [9] have adapted EF games to MTL and shown a number of expressiveness results. In this paper, we extend MTL EF games with counting moves corresponding to the $\mathrm{C}$ and UT modalities. We use the resulting $\mathrm{EF}$ theorem to characterise expressive powers of several fragments of CTMTL.

One attraction of pointwise MTL over finite timed words is that its satisfiability is decidable [7] whereas continuous time MTL has undecidable satisfiability. As our second main result, we show that MTL extended with $C$ and UT modalities also has decidable satisfiability. In order to prove this result, we give an equisatisfiable reduction from CTMTL to MTL. The reduction makes use of the notion of temporal projections modulo oversampling introduced earlier [3] where timed words satisfying original CTMTL formula have to be oversampled with additional time points to satisfy corresponding MTL formula. This result marks one more use of the technique of temporal projections. We note that our reduction can also be applied to MITL (with both $U$ and S) extended with $C$ and UT and it it gives an equisatisfiable formula in MITL which is exponential in the size of original formula. Thus, we establish that CTMITL [ U, S] has elementary satisfiability.

\section{A Zoo of Timed Temporal Logics}

In this section, we present the syntax and semantics of the various timed temporal logics we study in this paper. Let $\Sigma$ be a finite set of propositions. A finite timed word over $\Sigma$ is a tuple $\rho=(\sigma, \tau) . \sigma$ and $\tau$ are sequences $\sigma_{1} \sigma_{2} \ldots \sigma_{n}$ and $t_{1} t_{2} \ldots t_{n}$ respectively, with $\sigma_{i} \in 2^{\Sigma}-\emptyset$, and $t_{i} \in \mathbb{R}_{\geq 0}$ for $1 \leq i \leq n$ and $\forall i \in \operatorname{dom}(\rho), t_{i} \leq t_{i+1}$, where $\operatorname{dom}(\rho)$ is the set of positions $\{1,2, \ldots, n\}$ in the timed word. An example of a timed word over $\Sigma=\{a, b\}$ is $\rho=(\{a, b\}, 0.3)(\{b\}, 0.7)(\{a\}, 1.1) . \rho$ is strictly monotonic iff $t_{i}<t_{i+1}$ for all $i, i+1 \in \operatorname{dom}(\rho)$. Otherwise, it is weakly monotonic. The set of finite timed words over $\Sigma$ is denoted $T \Sigma^{*}$.

The logic MTL extends linear temporal logic (LTL) by adding timing constraints to the "until" modality of LTL. We parametrize this logic by a permitted set of time intervals denoted by $I \nu$. The intervals in $I \nu$ can be open, half-open or closed, with end 
points in $\mathbb{N} \cup\{0, \infty\}$. Such an interval is denoted $\langle a, b\rangle$. For example, $[3,7),[5, \infty)$. Let $t+\langle a, b\rangle=\langle t+a, t+b\rangle$.

\section{Metric Temporal Logic}

Given $\Sigma$, the formulae of MTL are built from $\Sigma$ using boolean connectives and time constrained version of the modality $\mathrm{U}$ as follows: $\varphi::=a(\in \Sigma)|\operatorname{true}| \varphi \wedge \varphi|\neg \varphi| \varphi \mathrm{U}_{I} \varphi$ where $I \in I \nu$. For a timed word $\rho=(\sigma, \tau) \in T \Sigma^{*}$, a position $i \in \operatorname{dom}(\rho)$, and an MTL formula $\varphi$, the satisfaction of $\varphi$ at a position $i$ of $\rho$ is denoted $(\rho, i) \models \varphi$, and is defined as follows:

$\rho, i \models a \leftrightarrow a \in \sigma_{i}$ and $\quad \rho, i \models \neg \varphi \leftrightarrow \rho, i \not \models \varphi$

$\rho, i \models \varphi_{1} \wedge \varphi_{2} \leftrightarrow \rho, i \models \varphi_{1}$ and $\rho, i \models \varphi_{2}$

$\rho, i \models \varphi_{1} \mathrm{U}_{I} \varphi_{2} \leftrightarrow \exists j>i, \rho, j \models \varphi_{2}, t_{j}-t_{i} \in I$, and $\rho, k \models \varphi_{1} \forall i<k<j$

$\rho$ satisfies $\varphi$ denoted $\rho \models \varphi$ iff $\rho, 1 \models \varphi$. Let $L(\varphi)=\{\rho \mid \rho, 1 \models \varphi\}$ denote the language of a MTL formula $\varphi$. Two formulae $\varphi$ and $\phi$ are said to be equivalent denoted as $\varphi \equiv \phi$ iff $L(\varphi)=L(\phi)$. Additional temporal connectives are defined in the standard way: we have the constrained future eventuality operator $\nabla_{I} a \equiv$ true $\mathrm{U}_{I} a$ and its dual $\square_{I} a \equiv \neg \diamond_{I} \neg a$. We also define the next operator as $\mathrm{O}_{I} \phi \equiv \perp \mathrm{U}_{I} \phi$. Weak versions of operators are defined as $\diamond_{I}^{\mathrm{w}} a=a \vee \diamond_{I} a, \square_{I}^{\mathrm{w}} a \equiv a \wedge \square_{I} a, a \cup_{I}^{\mathrm{w}} b \equiv b \vee\left[a \wedge\left(a \bigcup_{I} b\right)\right]$ if $0 \in I$, and $\left[a \wedge\left(a \mathrm{U}_{I} b\right)\right]$ if $0 \notin I$.

Theorem 1. Satisfiability checking of MTL is decidable over finite timed words and is non-primitive recursive. [7].

\section{Metric Temporal Logic with Counting (CTMTL)}

We denote by CTMTL the logic obtained by extending MTL with the ability to count, by endowing two counting modalities $\mathrm{C}$ as well as UT.

Syntax of CTMTL: $\varphi::=a(\in \Sigma) \mid$ true $|\varphi \wedge \varphi| \neg \varphi|\varphi| \mathrm{C}_{I}^{\geq n} \varphi \mid \varphi \mathrm{U}_{I, \eta} \varphi$, where $I \in I \nu, n \in \mathbb{N} \cup\{0\}$ and $\eta$ is a threshold formula of the form $\# \varphi \geq n$ or $\# \varphi<n$. The counting modality $\mathrm{C}_{\bar{I}}^{\geq n} \varphi$ is called the $\mathrm{C}$ modality, while $\varphi \mathrm{U}_{I, \eta} \varphi$ is called the UT modality. Let $\rho=(\sigma, \tau) \in T \Sigma^{*}, i, j \in \operatorname{dom}(\rho)$. Define

$N^{\rho}[i, I](\varphi)=\left\{k \in \operatorname{dom}(\rho) \mid t_{k} \in t_{i}+I \wedge \rho, k \models \varphi\right\}$, and $\rho[i, j](\varphi)=\{k \in \operatorname{dom}(\rho) \mid i<k<j \wedge \rho, k \models \varphi\}$.

Denote by $\left|N^{\rho}[i, I](\varphi)\right|$ and $|\rho[i, j](\varphi)|$ respectively, the cardinality of $N^{\rho}[i, I](\varphi)$ and $\rho[i, j](\varphi) .\left|N^{\rho}[i, I](\varphi)\right|$ is the number of points in $\rho$ that lie in the interval $t_{i}+I$, and which satisfy $\varphi$, while $|\rho[i, j](\varphi)|$ is the number of points lying between $i$ and $j$ which satisfy $\varphi$. Define $\rho, i \models \mathrm{C}_{\bar{I}}^{\geq n} \varphi$ iff $\left|N^{\rho}[i, I](\varphi)\right| \geq n$. Likewise, $\rho, i \models \varphi_{1} \mathrm{U}_{I, \# \varphi \geq n} \varphi_{2}$ iff $\exists j>i, \rho, j \models \varphi_{2}, t_{j}-t_{i} \in I$, and $\rho, k \models \varphi_{1}, \forall i<k<j$ and $|\rho[i, j](\varphi)| \geq n$.

Remark: The classical until operator of MTL is captured in CTMTL since $\varphi \mathrm{U}_{I} \psi \equiv$ $\varphi \mathrm{U}_{I, \# \text { true } \geq 0} \psi$. We can express $\mathrm{C}_{I}^{\sim n}$ and $\# \varphi \sim n$ for $\sim \in\{\leq,<,>,=\}$ in CTMTL since $\mathrm{C}_{I}^{<n} \varphi \equiv \neg \mathrm{C}_{\bar{I}}^{\geq n} \varphi, \mathrm{C}_{I}^{>n} \varphi \equiv \mathrm{C}_{\bar{I}}^{\geq n+1} \varphi, \mathrm{C}_{I}^{\leq n} \varphi \equiv \neg \mathrm{C}_{\bar{I}}^{\geq n+1} \varphi$ and $\# \varphi>n \equiv \# \varphi \geq$ $n+1, \# \varphi \leq n \equiv \neg(\# \varphi>n+1)$. Boolean combinations of threshold formulae are also expressible in CTMTL as shown by Lemmas 8 and 11 in Appendix B. Thus, $a \mathrm{U}_{(1,2), \# d=3 \wedge \# \mathrm{C}_{(0,1)}^{<2} \leq 5} c$ is expressible in CTMTL. The nesting depth of a CTMTL formula is the maximum nesting of C, UT operators. Formally, 
- $\operatorname{depth}\left(\varphi_{1} \mathrm{U}_{I, \# \varphi_{3} \sim n} \varphi_{2}\right)=\max \left(\operatorname{depth}\left(\varphi_{1}\right), \operatorname{depth}\left(\varphi_{2}\right), \operatorname{depth}\left(\varphi_{3}\right)+1\right)$,

$-\operatorname{depth}\left(C_{I}^{\geq n} \varphi\right)=\operatorname{depth}(\varphi)+1, \operatorname{depth}(\varphi \wedge \psi)=\max (\operatorname{depth}(\varphi), \operatorname{depth}(\psi))$,

- $\operatorname{depth}(\neg \varphi)=\operatorname{depth}(\varphi)$ and $\operatorname{depth}(a)=0$ for any $a \in \Sigma$.

For example, $\operatorname{depth}\left(a \mathrm{U}_{[0,2], \eta} \mathrm{C} \geq 1 b\right)$ with $\eta=\#\left[a \mathrm{U}_{(0,1), \#\left[\mathrm{C}_{(0,1)}^{=2} a \wedge \diamond_{(0,1), \# d=2}\right] \geq 1} c\right]<7$ is 3. We obtain the following natural fragments of CTMTL as follows: We denote by CMTL, the fragment of CTMTL obtained by using the $C$ modality and the $U_{I}$ modality. Further, $C^{0} \mathrm{MTL}$ denotes the subclass of CMTL where the interval $I$ in $\mathrm{C}_{I}^{n} \varphi$ is of the form $I=\langle 0, b\rangle$. When the interval is of the form $I=\langle 0,1\rangle$, then we denote the class by $C^{(0,1)}$ MTL. Note that $C^{(0,1)} M T L$ is the class which allows counting in the next one unit of time. This kind of counting (unit counting in future and past) was introduced and studied in [1] in the continuous semantics. $C^{(0,1)} \mathrm{MTL}$ is the pointwise counterpart of this logic, with only future operators. Clearly, $\mathrm{C}^{(0,1)} \mathrm{MTL} \subseteq \mathrm{C}^{0} \mathrm{MTL} \subseteq$ CMTL $\subseteq$ CTMTL. Restricting CTMTL to the UT modality, we obtain the fragment TMTL. Restricting the $\mathrm{C}$ modality to $\mathrm{C}^{(0,1)}$ or $\mathrm{C}^{0}$ and also allowing the UT modality, one gets the fragments $C^{(0,1)}$ TMTL and $C^{0} T M T L$ respectively. If we disallow the $C$ modality, restrict the intervals $I$ appearing in the formulae to non-punctual intervals of the form $\langle a, b\rangle(a \neq b)$, and restrict threshold formulae $\eta$ to be of the form \#true $\geq 0$, then we obtain MITL.

\section{Expressiveness Hierarchy in the Counting Zoo}

In this section, we study the expressiveness and hierarchy of the logics introduced in section 2. The main results of this section are the following:

Theorem 2. MTL $\subset \mathrm{C}^{(0,1)} \mathrm{MTL} \subset \mathrm{C}^{0} \mathrm{MTL} \subset \mathrm{TMTL}=\mathrm{C}^{0} \mathrm{TMTL} \subset \mathrm{CTMTL}$. Moreover, $\mathrm{CMTL}$ and $\mathrm{TMTL}$ are incomparable, and $\mathrm{C}^{0} \mathrm{MTL} \subset \mathrm{CMTL}$.

While Theorem 2 shows that there is an expressiveness gap between classical MTL and CTMTL, we show later that both these logics are equisatisfiable. Given $\varphi \in$ CTMTL, we can construct a formula $\psi \in$ MTL such that $\varphi$ is satisfiable iff $\psi$ is. Note that our notion of equisatisfiability is a special one modulo temporal projections. If $\varphi$ is over an alphabet $\Sigma, \psi$ is constructed over a suitable alphabet $\Sigma^{\prime} \supseteq \Sigma$ such that $L(\psi)$, when projected over to $\Sigma$ gives $L(\varphi)$.

Theorem 3. Satisfiability Checking of CTMTL is decidable over finite timed words.

The rest of this paper is devoted to the proofs of Theorems 2 and 3 , We establish Theorem 2 through Lemmas 1 to 4 To prove the separation between two logics, we define model-theoretic games.

\subsection{Model-Theoretic Games}

Our games are inspired from the standard model-theoretic games [13], [9]. The MTL games introduced in [9] can be found in Appendix C] We introduce CTMTL games. 
CTMTL Games Let $\left(\rho_{1}, \rho_{2}\right)$ be a pair of timed words. We define a $r$-round $k$-counting pebble $I_{\nu}$ game on $\left(\rho_{1}, \rho_{2}\right)$. The game is played on $\left(\rho_{1}, \rho_{2}\right)$ by two players, the Spoiler and the Duplicator. The Spoiler will try to show that $\rho_{1}$ and $\rho_{2}$ are $\{r, k\}$-distinguishable by some formula in CTMTL 3 while the Duplicator will try to show that $\rho_{1}, \rho_{2}$ are $\{r, k\}$-indistinguishable in TMTL. Each player has $r$ rounds and has access to a finite set of $\leq k$ pebbles from a box of pebbles $\mathcal{P}$ in each round of the game. Let $I_{\nu}$ be the set of permissible intervals allowed in the game.

A configuration of the game at the start of a round $p$ is a pair of points $\left(i_{p}, j_{p}\right)$ where $i_{p} \in \operatorname{dom}\left(\rho_{1}\right)$ and $j_{p} \in \operatorname{dom}\left(\rho_{2}\right)$. A configuration is called partially isomorphic, denoted $i \operatorname{sop}\left(i_{p}, j_{p}\right)$ iff $\sigma_{i_{p}}=\sigma_{j_{p}}$. Exactly one of the Spoiler or the Duplicator eventually wins the game. The initial configuration is $\left(i_{1}, j_{1}\right)$, the starting positions of both the words, before the first round. A 0 -round game is won by the Duplicator iff $i \operatorname{sop}\left(i_{1}, j_{1}\right)$. The $r$ round game is played by first playing one round from the starting position. Either the Spoiler wins the round, and the game is terminated or the Duplicator wins the round, and now the second round is played from this new configuration and so on. The Duplicator wins the game only if he wins all the rounds. The following are the rules of the game in any round. Assume that the current configuration is $\left(i_{p}, j_{p}\right)$.

- If $i \operatorname{sop}\left(i_{p}, j_{p}\right)$ is not true, then Spoiler wins the game, and the game is terminated. Otherwise, the game continues as follows:

- The Spoiler chooses one of the words by choosing $\rho_{x}, x \in\{1,2\}$. Duplicator has to play on the other word $\rho_{y}, x \neq y$. Then Spoiler plays either a $\mathrm{U}_{I, \eta}$ round, by choosing an interval $I \in I_{\nu}$, and a number $c \leq k$ of counting pebbles to be used, or a $\mathrm{C}_{I}^{c}$ round by choosing an interval $I \in I_{\nu}$ and a number $c \leq k$ of counting pebbles to be used. The number $c$ is obtained from $\eta=\# \varphi \geq c$ or $\eta=\neg(\# \varphi \geq c)$. $\mathrm{U}_{I, \eta}$ round: Given the current configuration as $\left(i_{p}, j_{p}\right)$ with $i \operatorname{sop}\left(i_{p}, j_{p}\right)$, then

- Spoiler chooses a position $i_{p}^{\prime} \in \operatorname{dom}\left(\rho_{x}\right)$ such that $i_{p}<i_{p}^{\prime}$ and $\left(t_{i_{p}^{\prime}}-t_{i_{p}}\right) \in I$.

- The Duplicator responds by choosing $j_{p}^{\prime} \in \operatorname{dom}\left(\rho_{y}\right)$ in the other word such that $j_{p}<j_{p}^{\prime}$ and $\left(t_{j_{p}^{\prime}}-t_{j_{p}}\right) \in I$. If the Duplicator cannot find such a position, the Spoiler wins the round and the game. Otherwise, the game continues and Spoiler chooses one of the following three options.

- $\diamond$ Part: The round ends with the configuration $\left(i_{p+1}, j_{p+1}\right)=\left(i_{p}^{\prime}, j_{p}^{\prime}\right)$.

- U Part: Spoiler chooses a position $j_{p}^{\prime \prime}$ in $\rho_{y}$ such that $j_{p}<j_{p}^{\prime \prime}<j_{p}^{\prime}$. The Duplicator responds by choosing a position $i_{p}^{\prime \prime}$ in $\rho_{x}$ such that $i_{p}<i_{p}^{\prime \prime}<i_{p}^{\prime}$. The round ends with the configuration $\left(i_{p+1}, j_{p+1}\right)=\left(i_{p}^{\prime \prime}, j_{p}^{\prime \prime}\right)$. If Duplicator cannot choose an $i_{p}^{\prime \prime}$, the game ends with Spoiler's win.

- Counting Part : First, Spoiler chooses one of the two words to play in the counting part. In his chosen word, Spoiler keeps $c \leq k$ pebbles from $\mathcal{P}$ at $c$ distinct positions between the points $j_{p}$ and $j_{p}^{\prime}$ (or $i_{p}$ and $i_{p}^{\prime}$ depending on the choice of the word). In response, the Duplicator also keeps $c$ pebbles from $\mathcal{P}$ at $c$ distinct positions between the points $i_{p}$ and $i_{p}^{\prime}\left(\right.$ or $j_{p}$ and $j_{p}^{\prime}$ ) in his word. Spoiler then chooses a pebbled position say $i_{p}^{\prime \prime}$ (note that $i_{p}<i_{p}^{\prime \prime}<i_{p}^{\prime}$ ) in the Duplicator's word. In response, Duplicator chooses a pebbled position $j_{p}^{\prime \prime}$

\footnotetext{
${ }^{3} \rho_{1}, \rho_{2}$ are $\{r, k\}$-distinguishable iff there exists a CTMTL formula $\varphi$ having $\operatorname{depth}(\varphi) \leq r$ with max counting constant $\leq k$ in any threshold formula $\eta$ or $\mathrm{C}$ modality in $\varphi$ such that $\rho_{1} \models \varphi$ and $\rho_{2} \not \models \varphi$ or vice-versa.
} 
(note that $j_{p}<j_{p}^{\prime \prime}<j_{p}^{\prime}$ ) in the Spoiler's word, and the game continues from the configuration $\left(i_{p+1}, j_{p+1}\right)=\left(i_{p}^{\prime \prime}, j_{p}^{\prime \prime}\right)$. At the end of the round, the pebbles are returned to the box of pebbles $\mathcal{P}$.

$\mathrm{C}_{I}^{\sim c}$ round: Given the current configuration as $\left(i_{p}, j_{p}\right)$ with $i \operatorname{sop}\left(i_{p}, j_{p}\right)$, Spoiler chooses an interval $I \in I_{\nu}$ as well as a number $c \leq k$. Spoiler then chooses one of the words to play (say $\rho_{1}$ ). From $i_{p}$, Spoiler places $c$ pebbles from $\mathcal{P}$ in the points lying in the interval $t_{i_{p}}+I$. In response, Duplicator also places $c$ pebbles from $\mathcal{P}$ in the points lying in $t_{j_{p}}+I$. Spoiler now picks a pebbled position $j_{p}^{\prime}$ in the word $\rho_{2}$, while Duplicator picks a pebbled position $i_{p}^{\prime}$ in the Spoiler's word. The round ends with the configuration $\left(i_{p}^{\prime}, j_{p}^{\prime}\right)$. At the end of the round, the pebbles are returned to the box of pebbles $\mathcal{P}$.

Intuition on Pebbling: To give some intuition behind the pebbling, consider $\# \varphi \geq c$ or $\mathrm{C}_{\bar{I}}^{\geq c} \varphi$. The idea behind Spoiler keeping $c$ pebbles on his word in the chosen interval $I$ is to say that these are the $c$ points where $\varphi$ evaluates to true. Duplicator is expected to find $c$ such points in his word. If Spoiler suspects that in the Duplicator's word, there are $<c$ positions in $I$ where $\varphi$ holds good, he picks up the appropriate pebble at the position where $\varphi$ fails. However, any pebbled position in Spoiler's word will satisfy $\varphi$. In this case, Duplicator loses. Similarly, if we have $\neg(\# \varphi \geq c)$, or $C_{I}^{<c} \varphi$, then Spoiler chooses the word (say $\rho_{1}$ ) on which $\varphi$ evaluates to true $\geq c$ times. Then Duplicator is on $\rho_{2}$. The idea is for Spoiler to find if there exist $c$ or more positions in the interval $I$ in $\rho_{1}$ where $\varphi$ holds good, and if so, pebble those points. This is based on Spoiler's suspicion that there are atleast $c$ positions in $I$ where $\varphi$ evaluates to true, violating the formula. In response, Duplicator does the same on $\rho_{2}$. Spoiler will now pick any one of the $c$ pebbles from $\rho_{2}$ and check for $\neg \varphi$. This is again based on Spoiler's belief that whichever $c$ points Duplicator pebbles in $\rho_{2}, \neg \varphi$ will evaluate to true in atleast one of them. If $\varphi$ holds at all the $c$ points in $\rho_{1}$, then Duplicator will lose on picking any pebble from $\rho_{1}$.

- We can restrict various moves according to the modalities provided by the logic. For example, in a TMTL $\left[\diamond_{I}\right]$ game, the possible rounds are $\diamond_{I}$ and $\diamond_{I, \eta}$. A CMITL game has only $\mathrm{U}_{I}, \mathrm{C}_{I}^{\geq n}$ rounds, with $I_{\nu}$ containing only non-punctual intervals.

Game equivalence: $\left(\rho_{1}, i_{1}\right) \approx_{r, k, I_{\nu}}\left(\rho_{2}, j_{1}\right)$ iff for every $r$-round, $k$-counting pebble CTMTL game over the words $\rho_{1}, \rho_{2}$ starting from the configuration $\left(i_{1}, j_{1}\right)$, the Duplicator always has a winning strategy.

Formula equivalence: $\left(\rho_{1}, i_{1}\right) \equiv_{r, k, I_{\nu}}^{\mathrm{CTMT}}\left(\rho_{2}, j_{1}\right)$ iff for every CTMTL formula $\varphi$ of depth $\leq r$ having max counting constant $\leq k$ in the C, UT modalities, $\rho_{1}, i_{1} \models \varphi \Longleftrightarrow$ $\rho_{2}, j_{1} \models \varphi$. The proof of Theorem 4 can be found in Appendix D

Theorem 4. $\left(\rho_{1}, i_{1}\right) \approx_{r, k, I_{\nu}}\left(\rho_{2}, j_{1}\right)$ iff $\left(\rho_{1}, i_{1}\right) \equiv_{r, k, I_{\nu}}^{\mathrm{CTMTL}}\left(\rho_{2}, j_{1}\right)$

We now use these games to show the separation between various logics. For brevity, from here on, we omit $I_{\nu}$ from the notations $\equiv_{r, k, I_{\nu}}^{\mathrm{CTMTL}}, \equiv_{r, k, I_{\nu}}^{\mathrm{CMTL}}, \equiv_{r, k, I_{\nu}}^{\mathrm{TMTL}}$ and $\equiv_{r, I_{\nu}}^{\mathrm{MTL}}$.

Lemma 1. $C M T L-T M T L \neq \emptyset$

Proof. Consider the formula $\varphi=\mathrm{C}_{(1,2)}^{\geq 2} a \in \mathrm{CMTL}$. We show that for any choice of $n$ rounds and $k$ pebbles, we can find two words $\rho_{1}, \rho_{2}$ such that $\rho_{1} \models \varphi, \rho_{2} \not \models \varphi$, but $\rho_{1} \equiv_{n, k}^{\mathrm{TMTL}} \rho_{2}$. Both $\rho_{1}, \rho_{2}$ are over $\Sigma=\{a\}$. Let $0<\delta<\epsilon<\frac{1}{10^{10 n k}}$ and 


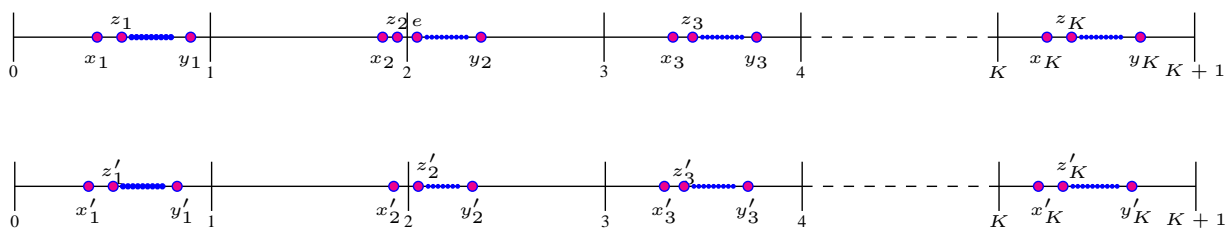

Fig. 1. Words showing CMTL $-T M T L \neq \emptyset$

$0<\kappa<\frac{\epsilon-\delta}{2 n k}$. Let $l$ be the maximum constant in $\mathbb{N}$ appearing in the permissible intervals $I_{\nu}$. Consider the word $\rho_{1}$ with $n l(k+1)=K$ unit intervals, with the following time stamps as depicted pictorially (Figure 1) and in the table.

\begin{tabular}{|c|c|c|}
\hline Points in & $\rho_{1}$ & $\rho_{2}$ \\
\hline$(0,1)$ & $\begin{array}{l}x_{1}=0.5, z_{1}=0.6, y_{1}=0.8 \\
\text { and } 2 n k \text { points between } z_{1}, y_{1} \\
\text { that are } \kappa \text { apart from each other }\end{array}$ & $\begin{array}{l}x_{1}^{\prime}=0.5, z_{1}^{\prime}=0.6, y_{1}^{\prime}=0.8 \\
\text { and } 2 n k \text { points between } z_{1}^{\prime}, y_{1}^{\prime} \\
\text { that are } \kappa \text { apart from each other }\end{array}$ \\
\hline$(1,2)$ & $x_{2}=1.8-\epsilon, z_{2}=1.8+\epsilon$ & $x_{2}^{\prime}=1.8-\epsilon$ \\
\hline$(2,3)$ & $\begin{array}{l}\quad e=2.4+n \epsilon, y_{2}=2.7+n \epsilon \\
\text { and } 2 n k \text { points between } e \text { and } y_{2} \\
\text { that are } \kappa \text { apart from each other }\end{array}$ & $\begin{array}{c}z_{2}^{\prime}=2.4+n \epsilon, y_{2}^{\prime}=2.7+n \epsilon \\
\text { and } 2 n k \text { points between } z_{2}^{\prime} \text { and } y_{2}^{\prime} \\
\text { that are } \kappa \text { apart from each other }\end{array}$ \\
\hline $\begin{array}{c}(i, i+1) \\
3 \leq i \leq K-1\end{array}$ & \begin{tabular}{|c|}
$x_{i}=i+0.4+(n-i) \epsilon$ \\
$z_{i}=i+0.8+(n+i) \epsilon+\delta$ \\
$y_{i}=i+0.8+(n+i+1) \epsilon$ and $2 n k$ points \\
between $z_{i}, y_{i}$ that are $\kappa$ apart from each other
\end{tabular} & $\begin{array}{c}x_{i}^{\prime}=i+0.4+(n-i) \epsilon \\
z_{i}^{\prime}=i+0.8+(n+i) \epsilon+\delta \\
y_{i}^{\prime}=i+0.8+(n+i+1) \epsilon \text { and } 2 n k \text { points } \\
\text { between } z_{i}, y_{i} \text { that are } \kappa \text { apart from each other }\end{array}$ \\
\hline
\end{tabular}

Thus, $\rho_{1}$ and $\rho_{2}$ differ only in the interval $(1,2): \rho_{1}$ has two points in $(1,2)$, while $\rho_{2}$ has only one. Thus, $\rho_{1} \models \varphi, \rho_{2} \not \models \varphi$.

Let $\operatorname{seg}\left(i_{p}\right) \in\{0,1, \ldots, K\}$ denote the left endpoint of the left closed, right open unit interval containing the point $i_{p} \in \operatorname{dom}\left(\rho_{1}\right)$ or $\operatorname{dom}\left(\rho_{2}\right)$. Our segments are $[0,1),[1,2)$, $\ldots,[K, K+1)$. For instance, if the configuration at the start of the $p$ th round is $\left(i_{p}, j_{p}\right)$ with time stamps $(1.2,3)$, then $\operatorname{seg}\left(i_{p}\right)=1, \operatorname{seg}\left(j_{p}\right)=3$. The following lemma says that in any round of the game, Duplicator can either achieve the same segment in both the words, or ensure that the difference in the segments is atmost 1. Moreover, by the choice of the words, there are sufficiently many segments on the right of any configuration so that Duplicator can always duplicate Spoiler's moves for the remaining rounds, preserving the lag of one segment.

Copy-cat strategy Consider the $p$ th round of the game with configuration $\left(i_{p}, j_{p}\right)$. If Duplicator can ensure that $\operatorname{seg}\left(i_{p+1}\right)-\operatorname{seg}\left(i_{p}\right)=\operatorname{seg}\left(j_{p+1}\right)-\operatorname{seg}\left(j_{p}\right)$, then we say that Duplicator has adopted a copy-cat strategy in the $p$ th round. We prove the following proposition to argue Duplicator's win.

Proposition 1. For an $n$ round TMTL game over the words $\rho_{1}, \rho_{2}$, the Duplicator always has a winning strategy such that for any $1 \leq p \leq n$, if $\left(i_{p}, j_{p}\right)$ is the initial configuration of the $p^{\text {th }}$ round, then $\left|\operatorname{seg}\left(i_{p}\right)-\operatorname{seg}\left(j_{p}\right)\right| \leq 1$. Moreover, when 
$\left|\operatorname{seg}\left(i_{p}\right)-\operatorname{seg}\left(j_{p}\right)\right|=1$, then there are atleast $(n-p)(l+1)$ segments to the right on each word after $p$ rounds, for all $1 \leq p \leq n$.

Proof. The initial configuration has time stamps $(0,0)$. We will play a $(n, k)$-TMTL game on $\rho_{1}, \rho_{2}$. Assume that the Spoiler chooses $\rho_{1}$ while the Duplicator chooses $\rho_{2}$. Since the interval $[1,2]$ is the only one different in both the words, it is interesting to look at the moves where the Spoiler chooses a point in interval $(1,2)$. We consider the two situations possible for Spoiler to land up in a point in interval $(1,2)$ : he can enter interval $(1,2)$ from some point in interval $(0,1)$, or directly choose to enter interval $(1,2)$ from the initial configuration with time stamps $(0,0)$.

Situation 1: Consider the case when from the starting configuration $\left(i_{1}, j_{1}\right)$ with time stamps $(0,0)$, Spoiler chooses a $\bigcup_{(1,2) \# a \sim c}$ move in $\rho_{1}$ and lands up at the point $x_{2}$ or $z_{2}$. In response, Duplicator has to come at the point $x_{2}^{\prime}$ in $\rho_{2}^{\prime}$. If $\left(i_{1}^{\prime}, j_{1}^{\prime}\right)$ has time stamps $\left(x_{2}, x_{2}^{\prime}\right)$ and if Spoiler chooses to pebble between 0 and $x_{2}$, then Duplicator pebbles between 0 and $x_{2}^{\prime}$; however, an identical configuration is obtained. Note that if Spoiler pebbles $\rho_{2}$, then Duplicator has it easy, since he will pebble the same positions in $\rho_{1}$. Let us hence consider obtaining the configuration $\left(i_{1}^{\prime}, j_{1}^{\prime}\right)$ with time stamps $\left(z_{2}, x_{2}^{\prime}\right)$, and let Spoiler pebble $\rho_{1}$. Spoiler can keep a maximum of $k$ pebbles in the points $x_{1}, \ldots, y_{1}, x_{2}$, while Duplicator keeps the same number of pebbles on the points $x_{1}^{\prime}, \ldots, y_{1}^{\prime}$. In this case, Spoiler has to a pick a pebbled position from among $x_{1}^{\prime}, \ldots, y_{1}^{\prime}$. In response, Duplicator will pick the same position from Spoiler's word and achieve an identical configuration. An interesting special case is when Spoiler keeps a single pebble at $x_{2}$ in $\rho_{1}$. In this case, Duplicator's best choice is to keep his pebble at $x_{1}^{\prime}$, so that the next configuration $\left(i_{2}, j_{2}\right)$ is one with time stamps $\left(x_{2}, x_{1}^{\prime}\right)$. $x_{1}^{\prime}$ and $x_{2}$ are topologically similar in the sense that the distribution of points in subsequent segments have some nice properties as given below.

Topological Similarity of Words: Consider the $2 n k+3$ points $x_{j}<z_{j}<p_{j}^{1}<\cdots<$ $p_{j}^{2 n k}<y_{j}$ in $\rho_{1}$, and $x_{j-1}^{\prime}<z_{j-1}^{\prime}<q_{j-1}^{1}<\ldots<q_{j-1}^{2 n k}<y_{j-1}^{\prime}$ in $\rho_{2}$, for $j \in\{2,3,4, \ldots, K\}$. Define a function $f$ that maps points in $\rho_{1}$ to topologically similar points in $\rho_{2}$.

$f:\left\{x_{j}, z_{j}, p_{j}^{1}, \ldots, p_{1}^{2 n k}, y_{j}\right\} \rightarrow\left\{x_{j-1}^{\prime}, z_{j-1}^{\prime}, q_{j-1}^{1}, \ldots, q_{j-1}^{2 n k}, y_{j-1}^{\prime}\right\}$ by $f\left(x_{j}\right)=x_{j-1}^{\prime}, f\left(z_{j}\right)=z_{j-1}^{\prime}, f\left(y_{j}\right)=y_{j-1}^{\prime}, f\left(p_{j}^{i}\right)=q_{j-1}^{i}$. Let $g=f^{-1}$.

(a) The current configuration has timestamps $\left(x_{2}, x_{1}^{\prime}\right)=\left(x_{2}, f\left(x_{2}\right)\right)$. For $j \geq 2$, if Spoiler chooses to move to any $p \in\left\{z_{j}, y_{j}, x_{j+2}\right\}$ from $x_{2}$, then Duplicator can move to $f(p)$ from $f\left(x_{2}\right)$ since, for any time interval $I$, it can be seen that $p-x_{2} \in I$ iff $f(p)-f\left(x_{1}\right) \in I$. Moreover, if Spoiler chooses to move to $x_{3}$ from $x_{2}$, then Duplicator can move to $z_{2}^{\prime}$ from $f\left(x_{2}\right)$ since, $x_{3}-x_{2}, z_{2}^{\prime}-f\left(x_{2}\right) \in(0,1)$.

(b) We can extend (a) above as follows: Let the current configuration have timestamps $(p, f(p))$ or $\left(x_{3}, z_{2}^{\prime}\right)$. Then it can be seen that for any $q \in\left\{x_{j}, y_{j}, z_{j}\right\}$ and interval $I, q-p \in I$ iff $f(q)-f(p) \in I$, and $q-x_{3} \in I$ iff $f(q)-z_{2}^{\prime} \in I$.

The facts claimed in (a) and (b) are evident from the construction of the timed words. They show that from a configuration $\left(i_{p}, j_{p}\right)$, such that $\operatorname{seg}\left(i_{p}\right)-\operatorname{seg}\left(j_{p}\right) \leq 1$, Duplicator can always achieve an intermediate configuration $\left(i_{p}^{\prime}, j_{p}^{\prime}\right)$ in any $\mathrm{U}_{I, \# a \sim c}$ such that $\operatorname{seg}\left(i_{p}^{\prime}\right)-\operatorname{seg}\left(j_{p}^{\prime}\right) \leq 1$. If Spoiler does not go for the until round or the 
counting round, then $\left(i_{p+1}, j_{p+1}\right)=\left(i_{p}^{\prime}, j_{p}^{\prime}\right)$. If Spoiler pebbles the points between $i_{p}$ and $i_{p}^{\prime}$ (or $j_{p}$ and $j_{p}^{\prime}$ ), then Duplicator can always ensure that he pebbles points $f(P)$ in $\rho_{2}$ whenever Spoiler pebbles a set of points $P$ in $\rho_{1}$. As a result, if Spoiler chooses a point $q=f(i) \in f(P)$ in $\rho_{2}$, then Duplicator can choose the point $g(q)=i \in P$ achieving the configuration $\left(i_{p+1}, j_{p+1}\right)=(g(q), q)=(i, f(i))$. By definition of $f, g$, we have $i_{p+1}-j_{p+1} \leq 1$. Note that Duplicator can also achieve an identical configuration if Spoiler moves ahead by several segments from $i_{p}$ (thus, $i_{p}^{\prime}>>i_{p}$ ), and pebbles a set of points that are also present between $j_{p}$ and $j_{p}^{\prime}$.

Situation 2: Starting from $\left(i_{1}, j_{1}\right)$ with time stamps $(0,0)$, if the Spoiler chooses a $\mathrm{U}_{(0,1), \# a \sim c}$ move and lands up at some point between $x_{1}$ and $y_{1}$, Duplicator will play copy-cat and achieve an identical configuration. Consider the case when Spoiler lands up at $\left.y_{1}\right]^{4}$. In response, Duplicator moves to $y_{1}^{\prime}$. From configuration $\left(i_{2}, j_{2}\right)$ with time stamps $\left(y_{1}, y_{1}^{\prime}\right)$, consider the case when Spoiler initiates a $\mathbf{U}_{(1,2), \# a \sim c}$ and moves to $z_{2}=1.8+\epsilon<2$. In response, Duplicator moves to the point $z_{2}^{\prime}=2.1>2$. A pebble is kept at the inbetween positions $x_{2}, x_{2}^{\prime}$ respectively in $\rho_{1}, \rho_{2}$. When Spoiler picks the pebble in Duplicator's word, then we obtain the configuration $\left(i_{3}, j_{3}\right)$ with time stamps $\left(x_{2}, x_{2}^{\prime}\right)$. If Spoiler does not get into the counting part/until part, the configuration obtained has time stamps $\left(z_{2}, z_{2}^{\prime}\right)$, with the lag of one segment $\left(\operatorname{seg}\left(i_{3}\right)=1, \operatorname{seg}\left(j_{3}\right)=2, \operatorname{seg}\left(j_{3}\right)-\operatorname{seg}\left(i_{3}\right)=1\right)$. We show in Appendix E that from $\left(i_{3}, j_{3}\right)$ with time stamps either $\left(x_{2}, x_{2}^{\prime}\right)$ or $\left(z_{2}, z_{2}^{\prime}\right)$, Duplicator can either achieve an identical configuration, or achieve a configuration with a lag of one segment.

From situations (1), (2) in Proposition 1] we know that either Duplicator achieves an identical configuration, in which case there is no segment lag, or there is a lag of atmost one segment. The length of the words are $l n k+n l=K$. If Spoiler always chooses bounded intervals (of length $\leq l$ ), then Duplicator respects his segment lag of 1 , and the maximum number of segments that can be explored in either word is atmost $n l<K$. In this case, after $p$ rounds, there are atleast $K-p l \geq n l k+n l-p l \geq$ $(n-p)(l+1)$ segments to the right of $\rho_{1}$ and $K-p l+1$ segments to the right of $\rho_{2}$. If Spoiler chooses an unbounded interval in any round, then Duplicator can either enforce an identical configuration in both situations 1 and 2 , or obtain one of the configurations with time stamps $(p, f(p)), f(p) \neq x_{2}^{\prime}$, or $\left(z_{2}, x_{2}^{\prime}\right)$ or $\left(x_{2}, x_{2}^{\prime}\right)$, from where it is known that Duplicator wins.

Lemma 2. $M T L \subset C^{(0,1)} M T L \subset C^{0} M T L$

Proof. We show that the formula $\varphi=\mathrm{C}_{(0,1)}^{=2} a \in \mathrm{C}^{(0,1)} \mathrm{MTL}$ cannot be expressed in MTL. Likewise, the formula $\varphi=\mathrm{C}_{(0,2)}^{\geq 2} a \in \mathrm{C}^{0} \mathrm{MTL}$ cannot be expressed in $\mathrm{C}^{(0,1)} \mathrm{MTL}$. A detailed proof of these are given by Propositions 2 and 3 in Appendix $\mathrm{F}$

Lemma 3. (i) $\mathrm{C}^{0} \mathrm{MTL} \subset \mathrm{TMTL}=\mathrm{C}^{0} \mathrm{TMTL}=\mathrm{C}^{(0,1)} \mathrm{TMTL}$ and (ii) $\mathrm{C}^{0} \mathrm{MTL} \subset \mathrm{CMTL}$.

Proof. (i) The first containment as well as the last two equalities follows from the fact that the counting modality $\mathrm{C}_{\langle 0, j\rangle}^{\geq n} \varphi$ of $\mathrm{C}^{0} \mathrm{MTL}$ can be written in TMTL as $\nabla_{\langle 0, j\rangle, \# \varphi \geq n}$ true.

\footnotetext{
${ }^{4}$ The argument when Spoiler lands up at $x_{1}$ or a point in between $x_{1}, y_{1}$ is exactly the same
} 
The strict containment of $C^{0} \mathrm{MTL}$ then follows from Lemma 4 (ii) We know that $\mathrm{C}^{0} \mathrm{MTL} \subseteq \mathrm{CMTL}$. This along with (i) and Lemma1 gives the strict containment.

Lemma 4. $T M T L-C M T L \neq \emptyset$

Proof. Consider the formula $\varphi=\bigotimes_{(0,1), \# a \geq 3} b \in \mathrm{TMTL}$. We show that for any choice of $n$ rounds and $k$ pebbles, we can find two words $\rho_{1}, \rho_{2}$ such that $\rho_{2} \models \varphi, \rho_{1} \not \models \varphi$, but $\rho_{1} \equiv_{n, k}^{\mathrm{CMTL}} \rho_{2}$. The words can be seen in Figure 2 and the details in Appendix $\mathrm{G}$

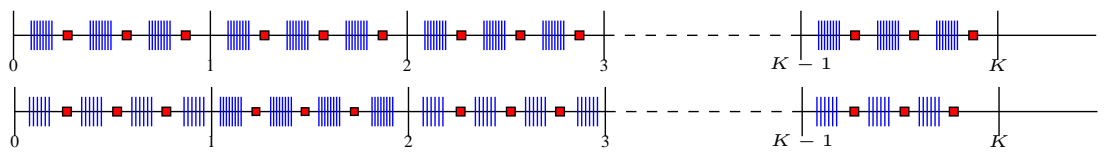

Fig. 2. The red square represents $a$, the bunch of blue lines represents a bunch of $b$ 's. There are 3 $a$ 's in each unit interval of both $\rho_{1}$ and $\rho_{2}$. The difference is that $\rho_{1}$ has 3 blocks of $b$ 's in each unit interval, while $\rho_{2}$ has 4 blocks of $b$ 's in each unit interval except the last. Clearly, $\rho_{2} \models \varphi, \rho_{1} \not \models \varphi$.

\section{Satisfiability Checking of Counting Logics}

In this section, we show that CTMTL has a decidable satisfiability checking. For this, given a formula in CTMTL we synthesize an equisatisfiable formula in MTL, and use the decidability of MTL. We start discussing some preliminaries. Let $\Sigma, X$ be finite sets of propositions such that $\Sigma \cap X=\emptyset$.

1. $(\Sigma, X)$-simple extensions. A $(\Sigma, X)$-simple extension is a timed word $\rho^{\prime}=\left(\sigma^{\prime}, \tau^{\prime}\right)$ over $X \cup \Sigma$ such that at any point $i \in \operatorname{dom}\left(\rho^{\prime}\right), \sigma_{i}^{\prime} \cap \Sigma \neq \emptyset$. For $\Sigma=\{a, b\}, X=$ $\{c, d\},(\{a\}, 0.2)(\{b, c, d\}, 0.3)(\{b, d\}, 1.1)$ is a $(\Sigma, X)$-simple extension. However, $(\{a\}, 0.2)(\{c, d\}, 0.3)(\{b, d\}, 1.1)$ is not.

2. Simple Projections. Consider a $(\Sigma, X)$-simple extension $\rho$. We define the simple projection of $\rho$ with respect to $X$, denoted $\rho \backslash X$ as the word obtained by erasing the symbols of $X$ from each $\sigma_{i}$. Note that $\operatorname{dom}(\rho)=\operatorname{dom}(\rho \backslash X)$. For example, if $\Sigma=\{a, c\}, X=\{b\}$, and $\rho=(\{a, b, c\}, 0.2)(\{b, c\}, 1)(\{c\}, 1.3)$, then $\rho \backslash X=$ $(\{a, c\}, 0.2)(\{c\}, 1)(\{c\}, 1.3) . \rho \backslash X$ is thus, a timed word over $\Sigma$. If the underlying word $\rho$ is not a $(\Sigma, X)$-simple extension, then $\rho \backslash X$ is undefined.

3. $(\Sigma, X)$-oversampled behaviours. $\mathrm{A}(\Sigma, X)$-oversampled behaviour is a timed word $\rho^{\prime}=\left(\sigma^{\prime}, \tau^{\prime}\right)$ over $X \cup \Sigma$, such that $\sigma_{1}^{\prime} \cap \Sigma \neq \emptyset$ and $\sigma_{\mid \operatorname{dom}\left(\rho^{\prime}\right) !}^{\prime} \cap \Sigma \neq \emptyset$. Oversampled behaviours are more general than simple extensions since they allow occurrences of new points in between the first and the last position. These new points are called oversampled points. All other points are called action points. For $\Sigma=\{a, b\}, X=\{c, d\},(\{a\}, 0.2)(\{c, d\}, 0.3)(\{a, b\}, 0.7)(\{b, d\}, 1.1)$ is a $(\Sigma, X)$-oversampled behaviour, while $(\{a\}, 0.2)(\{c, d\}, 0.3)(\{c\}, 1.1)$ is not. 
4. Oversampled Projections. Given a $(\Sigma, X)$-oversampled behaviour $\rho^{\prime}=\left(\sigma^{\prime}, \tau^{\prime}\right)$, the oversampled projection of $\rho^{\prime}$ with respect to $\Sigma$, denoted $\rho^{\prime} \downarrow X$ is defined as the timed word obtained by deleting the oversampled points, and then erasing the symbols of $X$ from the action points. $\rho=\rho^{\prime} \downarrow X$ is a timed word over $\Sigma$.

A temporal projection is either a simple projection or an oversampled projection. We now define equisatisfiability modulo temporal projections. Given MTL formulae $\psi$ and $\phi$, we say that $\phi$ is equisatisfiable to $\psi$ modulo temporal projections iff there exist disjoint sets $X, \Sigma$ such that (1) $\phi$ is over $\Sigma$, and $\psi$ over $\Sigma \cup X$, (2) For any timed word $\rho$ over $\Sigma$ such that $\rho \models \phi$, there exists a timed word $\rho^{\prime}$ such that $\rho^{\prime} \models \psi$, and $\rho$ is a temporal projection of $\rho^{\prime}$ with respect to $X$, (3) For any behaviour $\rho^{\prime}$ over $\Sigma \cup X$, if $\rho^{\prime} \models \psi$ then the temporal projection $\rho$ of $\rho^{\prime}$ with respect to $X$ is well defined and $\rho \models \phi$.

If the temporal projection used above is a simple projection, we call it equisatisfiability modulo simple projections and denote it by $\phi=\exists X$. $\psi$. If the projection in the above definition is an oversampled projection, then it is called equisatisfiability modulo oversampled projections and is denoted $\phi \equiv \exists \downarrow X$. $\psi$. Equisatisfiability modulo simple projections are studied extensively [12], [10], [4]. It can be seen that if $\varphi_{1}=\exists X_{1} \cdot \psi_{1}$ and $\varphi_{2}=\exists X_{2} . \psi_{2}$, with $X_{1}, X_{2}$ disjoint, then $\varphi_{1} \wedge \varphi_{2}=\exists\left(X_{1} \cup X_{2}\right) .\left(\psi_{1} \wedge \psi_{2}\right)$ [6].

As in the case of simple projections, equisatisfiability modulo oversampled projections are also closed under conjunctions when one considers the relativized formulae. For example, consider a formula $\varphi=\square_{(0,1)} a$ over $\Sigma=\{a, d\}$. Let $\psi_{1}=\square_{(0,1)}(a \vee b) \wedge$ $\diamond_{(0,1)}(b \wedge \neg a)$ be a formula over the extended alphabet $\{a, b, d\}$ and $\psi_{2}=\square(c \leftrightarrow$ $\left.\square_{(0,1)} a\right) \wedge c$ over the extended alphabet $\{a, c, d\}$. Note that $\varphi=\exists \downarrow\{b\} . \psi_{1}$ and $\varphi=\exists \downarrow\{c\} . \psi_{2}$ but $\varphi \wedge \varphi \neq \exists \downarrow\{b, c\} .\left(\psi_{1} \wedge \psi_{2}\right)$ as the left hand side evaluates to $\varphi$ which is satisfiable while the right hand side is unsatisfiable. This is due to the presence of a non-action point where only $b$ holds. But this can easily be fixed by relativizing all the formulae over their respective action points. $\psi_{1}$ is relativized as $\lambda_{1}=\square_{(0,1)}\left(a_{c t} \rightarrow(a \vee b)\right) \wedge \diamond_{(0,1)}\left(a c t_{1} \wedge b \wedge \neg a\right)$ and $\psi_{2}$ is relativized as $\lambda_{2}=\square\left(a c t_{2} \rightarrow\left(c \leftrightarrow \square_{(0,1)}\left(a c t_{2} \rightarrow a\right)\right)\right) \wedge a c t_{2} \wedge c$ where $a c t_{1}=b \vee d \vee a$ and $a c t_{2}=a \vee c \vee d$. Now, $\varphi \wedge \varphi=\exists \downarrow\{b, c\} .\left(\lambda_{1} \wedge \lambda_{2}\right)$. The relativized forms of $\psi_{1}, \psi_{2}$ are called their Oversampled Normal Forms with respect to $\Sigma$ and denoted $O N F_{\Sigma}\left(\psi_{1}\right)$ and $O N F_{\Sigma}\left(\psi_{2}\right)$. Then it can be seen that $\varphi_{1} \wedge \varphi_{2}=\exists \downarrow\{b, d\}$. $\left[O N F_{\Sigma}\left(\psi_{1}\right) \wedge O N F_{\Sigma}\left(\psi_{2}\right)\right]$, and $\varphi_{1}=\exists \downarrow\{b\} . O N F_{\Sigma}\left(\psi_{1}\right), \varphi_{2}=\exists \downarrow\{d\} . O N F_{\Sigma}\left(\psi_{2}\right)$. The formal definition of $O N F_{\Sigma}(\varphi)$ for a formula $\varphi$ over $\Sigma \cup X$ can be found in Appendix $\mathbf{H}$. Equisatisfiability modulo oversampled projections were first studied in [3] to eliminate non-punctual past from MTL over timed words. We use equisatifiability modulo simple projections to eliminate the $\mathrm{C}$ modality and oversampled projections to eliminate the UT modality from CTMTL.

\section{Elimination of Counting Modalities from CTMTL}

In this section, we show how to eliminate the counting constraints from CTMTL over strictly monotonic timed words. This can be extended to weakly monotonic timed words.

Given any CTMTL formula $\varphi$ over $\Sigma$, we "flatten" the C, UT modalities of $\varphi$ and obtain a flattened formula. As an example, consider the formula $\varphi=a \mathrm{U}_{[0,3]}(c \wedge$ 
$\left.\left.\mathrm{C}_{(2,3)}^{=1} d \mathrm{U}_{(0,1), \#\left(d \wedge \mathrm{C}_{(0,1)}^{=1} e\right) \geq 1} \mathrm{C}_{(0,1)}^{\geq 2} e\right]\right)$. Replacing the counting modalities with fresh witness propositions $w_{1}, w_{2}$, we obtain $\varphi_{\text {flat }}=\left[a \mathrm{U}_{[0,3]}\left(c \wedge w_{1}\right)\right] \wedge T$ where $T=$ $T_{1} \wedge T_{2} \wedge T_{3} \wedge T_{4}$, with $\left.T_{1}=\square^{\mathrm{w}}\left[w_{1} \leftrightarrow \mathrm{C}_{(2,3)}^{=1} w_{2}\right], T_{2}=\square^{\mathrm{w}}\left[w_{2} \leftrightarrow d \mathrm{U}_{(0,1), \# w_{4} \geq 1} w_{3}\right]\right]$, $T_{3}=\square^{\mathrm{w}}\left[w_{4} \leftrightarrow\left(d \wedge \mathrm{C}_{(0,1)}^{=1} e\right)\right]$, and $T_{4}=\square^{\mathrm{w}}\left[w_{3} \leftrightarrow \mathrm{C}_{(0,1)}^{\geq 2} e\right]$. Each temporal projection $T_{i}$ obtained after flattening contains either a $\mathrm{C}$ modality or a UT modality. In the following, we now show how to obtain equisatisfiable MTL formulae corresponding to each temporal projection. The proof of Lemma 5 is in Appendix []

Lemma 5. The formula $\mathrm{C}_{\langle l, \infty)}^{\geq n}$ b has an equivalent formula in MTL.

We now outline the steps followed to obtain an equisatisfiable formula in MTL, assuming $\mathrm{C}_{\langle l, \infty)}^{\geq n} b$ modalities have been eliminated using Lemma 5 .

1. Flattening : Flatten $\chi$ obtaining $\chi_{\text {flat }}$ over $\Sigma \cup W$, where $W$ is the set of witness propositions used, $\Sigma \cap W=\emptyset$.

2. Eliminate Counting: Consider, one by one, each temporal definition $T_{i}$ of $\chi_{\text {flat }}$. Let $\Sigma_{i}=\Sigma \cup W \cup X_{i}$, where $X_{i}$ is a set of fresh propositions, $X_{i} \cap X_{j}=\emptyset$ for $i \neq j$.

- If $T_{i}$ is a temporal projection containing a $\mathrm{C}$ modality of the form $\mathrm{C}_{\langle l, u\rangle}^{\sim n}$, or a UT modality of the form $x \mathrm{U}_{I, \# b \leq n} y$, then Lemma 6 synthesizes a formula $\zeta_{i} \in \mathrm{MTL}$ over $\Sigma_{i}$ such that $T_{i} \equiv \exists X_{i} \cdot \zeta_{i}$.

- If $T_{i}$ is a temporal projection containing a UT modality of the form $x \mathrm{U}_{I, \# b \geq n} y$, Lemma7gives $\zeta_{i} \in \mathrm{MTL}$ over $\Sigma_{i}$ such that $O N F_{\Sigma}\left(T_{i}\right) \equiv \exists \downarrow X_{i} \cdot \zeta_{i}$.

3. Putting it all together: The formula $\zeta=\bigwedge_{i=1}^{k} \zeta_{i} \in \mathrm{MTL}$ is such that $\bigwedge_{i=1}^{k} O N F_{\Sigma}\left(T_{i}\right) \equiv \exists \downarrow X . \bigwedge_{i=1}^{k} \zeta_{i}$ where $X=\bigcup_{i=1}^{k} X_{i}$.

Lemma 6. 1. Consider a temporal definition $T=\square^{\mathrm{w}}\left[a \leftrightarrow \mathrm{C}_{[l, u)}^{\geq n} b\right]$, built from $\Sigma \cup W$. Then we synthesize a formula $\zeta \in \mathrm{MTL}$ over $\Sigma \cup W \cup X$ such that $T \equiv \exists X . \zeta$.

2. Consider a temporal definition $T=\square^{\mathrm{w}}\left[a \leftrightarrow x \cup_{I, \# b \leq n} y\right]$, built from $\Sigma \cup W$. Then we synthesize a formula $\zeta \in \mathrm{MTL}$ over $\Sigma \cup W \cup X$ such that $T \equiv \exists X . \zeta$.

Proof. 1. Lets consider intervals of the form $[l, u)$. Our proof extends to all intervals $\langle l, u\rangle$. Consider $T=\square^{\mathrm{w}}\left[a \leftrightarrow \mathrm{C}_{[l, u)}^{\geq n} b\right]$. Let $\oplus$ denote addition modulo $n+1$.

(a) Construction of a $(\Sigma \cup W, X)$ - simple extension. We introduce a fresh set of propositions $X=\left\{b_{0}, b_{1}, \ldots, b_{n}\right\}$ and construct a simple extension $\rho^{\prime}=$ $\left(\sigma^{\prime}, \tau^{\prime}\right)$ from $\rho=(\sigma, \tau)$ as follows:

- $C 1: \sigma_{1}^{\prime}=\sigma_{1} \cup\left\{b_{0}\right\}$. If $b_{k} \in \sigma_{i}^{\prime}$ and if $b \in \sigma_{i+1}, \sigma_{i+1}^{\prime}=\sigma_{i+1} \cup\left\{b_{k \oplus 1}\right\}$.

- $C 2$ : If $b_{k} \in \sigma_{i}^{\prime}$ and $b \notin \sigma_{i+1}$, then $\sigma_{i+1}^{\prime}=\sigma_{i+1} \cup\left\{b_{k}\right\}$.

- C3: $\sigma_{i}^{\prime}$ has exactly one symbol from $X$ for all $1 \leq i \leq|\operatorname{dom}(\rho)|$.

(b) Formula specifying the above behaviour. The variables in $X$ help in counting the number of $b$ 's in $\rho . C 1$ and $C 2$ are written in MTL as follows:

$$
\text { - } \delta_{1}=\bigwedge_{k=0}^{n} \square^{\mathrm{w}}\left[\left(\mathrm{O} b \wedge b_{k}\right) \rightarrow \mathrm{O} b_{k \oplus 1}\right] \text { and } \delta_{2}=\bigwedge_{k=0}^{n} \square^{\mathrm{w}}\left[\left(\mathrm{O} \neg b \wedge b_{k}\right) \rightarrow \mathrm{O} b_{k}\right]
$$

(c) Marking the witness ' $a$ ' correctly at points satisfying $\mathrm{C}_{[l, u)}^{\geq n} b$. The index $i$ of $b_{i}$ at a chosen point gives the number of $b$ 's seen so far since the previous occurrence of $b_{0}$. From a point $i$, if the interval $\left[t_{i}+l, t_{i}+u\right)$ has $k$ elements of 
$X$, then there must be $k b$ 's in $\left[t_{i}+l, t_{i}+u\right)$. To mark the witness $a$ appropriately, we need to check the number of times $b$ occurs in $\left[t_{i}+l, t_{i}+u\right]$ from the current point $i$. A point $i \in \operatorname{dom}\left(\rho^{\prime}\right)$ is marked with witness $a$ iff all variables of $X$ are present in $\left[t_{i}+l, t_{i}+u\right)$, as explained in MTL by $\kappa=\square^{\mathrm{w}}\left[a \leftrightarrow\left(\bigwedge_{k=1}^{n} \nabla_{[l, u)} b_{k}\right)\right]$. $\zeta=\delta_{1} \wedge \delta_{2} \wedge \kappa$ in MTL is equisatisfiable to $T$ modulo simple projections.

2. The proof is similar to the above, details are in Appendix 』

Lemma 7. Consider a temporal definition $T=\square^{\mathrm{w}}\left[a \leftrightarrow x \mathrm{U}_{I, \# b \geq n} y\right]$, built from $\Sigma \cup W$. Then we synthesize a formula $\psi \in \mathrm{MTL}$ over $\Sigma \cup W \cup X$ such that $O N F_{\Sigma}(T) \equiv$ $\exists \downarrow X$. $\psi$ where $O N F_{\Sigma}(T)$ is $T$ relativized with respect to $\Sigma$.

Proof. If $I$ is of the form $\langle l, \infty)$, then $x \mathrm{U}_{\langle l, \infty), \# b \geq n} y \equiv x \mathrm{U}_{\langle l, \infty)} y \wedge x \mathrm{U}_{\# b \geq n} y$. The untimed threshold formula $x \mathrm{U}_{\# b \geq n} y$ can be rewritten in LTL [5].

The next case is when the interval $I$ is bounded of the form $[l, u)$. Our reduction below can be adapted to other kinds of bounded intervals. Let $j$ be any point. Let $f a r_{j}$ be the farthest point in the $[l, u)$ future of $j$ such that $y$ is true at $f_{a r}$, and $x$ continuously holds at all the intermediate points between $j$ and $f_{a r}$. To check the truth of $x \mathrm{U}_{I, \# b \geq n} y$ at $j$, we need to assert that the number of $b$ 's from $j$ to $f a r_{j}$ is $\geq n$. We first count the number of $b$ 's from the first integer point in the $[l, u)$ future of $j$ (let this point be $\alpha$ ) to $f a r_{j}$ and add this to the number of $b$ 's between $j$ and $\alpha$. In case $f a r_{j}$ lies before $\alpha$, then we simply count the number of $b$ 's between $j$ and $f a r_{j}$. Since we may not have all integer points at our disposal, we oversample the model by adding extra points at all integer time stamps.

Let $L=u-l$. Define $s \boxplus t=\min (s+t, n)$, and $s \oplus t=(s+t) \bmod (u+1)$. 1) Construction of a $(\Sigma \cup W, X)$-oversampled behaviour. We introduce a fresh set of propositions $X=C \cup A \cup B$ where $C, B, A$ are defined below. Given any timed word $\rho$, we then construct a $(\Sigma \cup W, X)$-oversampled behaviour $\rho^{\prime}=\left(\sigma^{\prime}, \tau^{\prime}\right)$ from $\rho=(\sigma, \tau)$.

- O1: $C=\left\{c_{0}, c_{1}, \ldots, c_{u}\right\}$. A point $i$ of $\rho$ is marked $c_{g}$ iff $t_{i} \bmod u=g$. In the absence of such a point $i$ (such that $t_{i}$ is an integer value $k<t_{|\operatorname{dom}(\rho)|}$ ), we add a new point $i$ to $\operatorname{dom}(\rho)$ with time stamp $t_{i}^{\prime}$ and mark it with $c_{g}$ iff $t_{i}^{\prime} \bmod u=g$. Let $\rho_{c}=\left(\sigma^{c}, \tau^{c}\right)$ denote the word obtained from $\rho$ after this marking.

- O2: $B=\cup_{i=0}^{u} B^{i}$, where $B^{i}=\left\{b_{0}^{i}, b_{1}^{i}, \ldots b_{n}^{i}\right\}$. All the points of $\rho_{c}$ marked $c_{i}$ are marked as $b_{0}^{i}$. Let $p, q$ be two integer points such that $p$ is marked $c_{i}, q$ is marked $c_{i \oplus L}$, and no point between $p, q$ is marked $c_{i \oplus L} \cdot p, q$ are $L$ apart from each other. Let $p<r<q$ be such that $b_{g}^{i} \in \sigma_{r}^{c}$ for some $g$. If $c_{i \oplus L} \notin \sigma_{r+1}^{c}$ and $b \in \sigma_{r+1}^{c}$, then the point $r+1$ is marked $b_{g \boxplus 1}^{i}$. If $c_{i \oplus L}, b \notin \sigma_{r+1}^{c}$, then the point $r+1$ is marked $b_{g}^{i}$. Each $B^{i}$ is a set of counters which are reset at $c_{i}$ and counts the number of occurrences of $b$ upto the threshold $n$ between a $c_{i}$ and the next occurrence of $c_{i \oplus L}$. Starting at a point marked $c_{i}$ with counter $b_{0}^{i}$, the counter increments upto $n$ on encountering a $b$, until the next $c_{i \oplus L}$. Further, we ensure that the counter $B^{i}$ does not appear anywhere from $c_{i \oplus L}$ to the next $c_{i}$. Let the resultant word be $\rho_{b}$.

- O3: $A=\left\{a_{0}, a_{1}, \ldots, a_{n}\right\}$. Consider any point $j$ in $\rho_{b}$ with time stamp $t_{j}$. Let $\alpha$ be a point with time stamp $\left\lceil t_{j}+l\right\rceil$. Let $\max _{j}$ represent a point satisfying the following conditions: (a) $y$ is true at $\max _{j}$ and $t_{\max _{j}} \in\left[t_{j}+l, t_{j}+u\right.$ ), (b) $x$ is true at all points between $j$ and $\max _{j}$, and (c) the number of occurrences of $b$ from $\alpha$ 


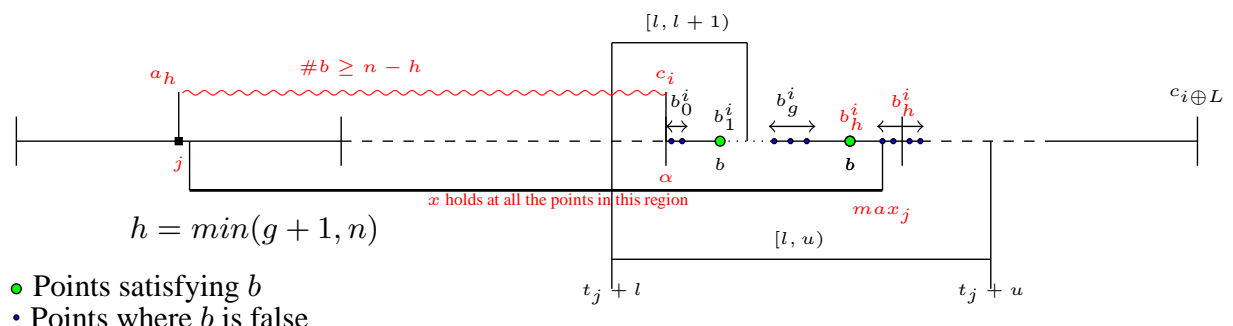

- Points where $b$ is false

Fig. 3. Illustration of point $j, \max _{j}$ and the point $\alpha$ such that $t_{\alpha}=\left\lceil t_{j}+l\right\rceil . \alpha$ is marked with some $c_{i}$ since it is an integer time point. The counting of $b$ 's is reset at $c_{i}$, starting with $b_{0}^{i}$, and continues till $c_{i \oplus L}$. Since $\max _{j}$ is marked $b_{h}^{i}, j$ is marked $a_{h}$. $h$ is the count of $b$ 's between $\alpha$ and $\max _{j}$. To satisfy $\square\left(a \leftrightarrow x \mathrm{U}_{[l, u), \# b \geq n} y\right)$ at $j$, we check that the number of $b$ 's between $j$ and $\alpha$ is $\geq n-h$ when $b$ is not true at $\alpha$, and is $\geq n-h-1$ when $b$ is true at $\alpha$.

to $\max _{j}$ is either $\geq n$, or is the maximum amongst all points which satisfy (a) and (b). The point $j$ is marked $a_{h}$ iff $h<n$ is the number of occurrences of $b$ 's from $\alpha$ to $\max _{j}$. If the count of $b$ 's from $\alpha$ to $\max _{j}$ is $\geq n$, then $j$ is marked $a_{n}$. Note that whenever $\max _{j}$ exists, it will be at or after $\alpha . \max _{j}$ need not always exist; we could have a point $\beta$ with time stamp $t_{j} \leq t_{\beta} \leq t_{\alpha}$ such that $y$ is true at $\beta, x$ holds continuously between $j$ and $\beta$, and the number of occurrences of $b$ in between $j$ and $\beta$ is $\geq n$. Let $\rho^{\prime}$ be the word obtained after all the markings.

2) Formula for specifying above behaviour. We give following $M T L$ formulae to specify $O 2$ and $O 3 . \delta_{2}=\bigwedge_{i=0}^{u}\left(\delta_{2 i}(1) \wedge \delta_{2 i}(2) \wedge \delta_{2 i}(3)\right)$ encodes $O 2$ where $\delta_{2 i}(1)=\square^{\mathrm{w}}\left(c_{i} \rightarrow b_{0}^{i}\right) \wedge \bigwedge_{k=0}^{n} \square^{\mathrm{w}}\left[\left(\mathrm{O}\left(b \wedge \neg c_{i \oplus L}\right) \wedge b_{k}^{i}\right) \rightarrow \mathrm{O} b_{k \boxplus 1}^{i}\right]$, $\delta_{2 i}(2)=\bigwedge_{k=0}^{n} \square^{\mathrm{w}}\left[\left(\mathrm{O}\left(\neg b \wedge \neg c_{i \oplus L}\right) \wedge b_{k}^{i}\right) \rightarrow \mathrm{O} b_{k}^{i}\right]$ and $\delta_{2 i}(3)=\bigwedge_{i=0}^{u} \square^{\mathrm{w}}\left[c_{i \oplus L} \rightarrow\left(\neg c_{i} \wedge \neg b^{i}\right) \mathrm{U} c_{i}\right]$, where $b^{i}=\bigvee_{k=0}^{u} b_{k}^{i}$. O3 is encoded by $\delta_{3}=\bigvee_{i=0}^{i=u}\left(\square^{\mathrm{w}}\left[a_{h} \leftrightarrow(\neg a c t \vee x) \mathrm{U}_{[l, u)}\left(y \wedge b_{h}^{i}\right) \wedge \neg\left\{(\neg a c t \vee x) \mathrm{U}_{[l, u)}\left(y \wedge b_{h+1}^{i}\right)\right\}\right] \wedge \diamond_{\mathcal{I}} c_{i}\right)$ where $\mathcal{I}=[l, l+1)$. The truth of $\delta_{3}$ relies on the fact that if $x \mathrm{U}_{[l, u)}\left(y \wedge b_{h}^{1}\right)$ and $x \mathrm{U}_{[l, u)}\left(y \wedge b_{h+2}^{i}\right)$ are both true at a point, then $x \mathrm{U}_{[l, u)}\left(y \wedge b_{h+1}^{i}\right)$ is also true at the same point. Hence, if $x \mathrm{U}_{[l, u)}\left(y \wedge b_{h}^{i}\right)$ is true, and $x \mathrm{U}_{[l, u)}\left(y \wedge b_{h+1}^{i}\right)$ is not true at some point, then $h$ is the largest number such that $x \mathrm{U}_{[l, u)}\left(y \wedge b_{h}^{i}\right)$ is true. Let act $=\bigvee(\Sigma \cup W)$.

3) Marking the witness ' $a$ ' correctly at points satisfying $x \mathrm{U}_{I, \# b \geq n} y$. Let $j$ be any point in $\rho^{\prime}$, such that $\max _{j}$ exists. We first count the number of $b$ 's from $j$ to the farthest integer point $\alpha$ (recall that $t_{\alpha}=\left\lceil t_{j}+l\right\rceil$ ), followed by counting the number of $b$ 's from $\alpha$ to $\max _{j}$. Note that the index $h$ of $a_{h}$ marked at $j$ gives the count (upto $n$ ) of $b$ 's from $\alpha$ to $\max _{j}$. We check the count of $b$ 's between $j$ and $\alpha$ is $\geq n-h$. Let $\mathcal{I}=[l, l+1)$. 


$$
\begin{aligned}
\lambda_{1} & =\bigvee_{h=0, i=0}^{h=n, i=u}\left[\left(a_{h} \wedge \diamond_{\mathcal{I}}\left(c_{i} \wedge \neg b\right) \wedge\left[(\neg a c t \vee x) \wedge \neg c_{i}\right] \mathrm{U}_{\# b \geq n-h} c_{i}\right)\right] \\
\lambda_{2} & =\bigvee_{h=0, i=0}^{h=n, i=u}\left[\left(a_{h} \wedge \diamond_{\mathcal{I}}\left(c_{i} \wedge b\right) \wedge\left[(\neg a c t \vee x) \wedge \neg c_{i}\right] \mathrm{U}_{\# b \geq n-h-1} c_{i}\right)\right]
\end{aligned}
$$

If $\max _{j}$ does not exist, then we characterize the point $\beta$ by the truth of the formula $\lambda_{3}=((x \vee \neg a c t) \wedge \neg c) \mathrm{U}_{\#_{b} \geq n} y$, where $c=\bigvee c_{k}$. The formula $\lambda=\square^{\mathrm{w}}[a \leftrightarrow$ $\left(\lambda_{1} \vee \lambda_{2} \vee \lambda_{3}\right)$ ] captures marking point $j$ correctly with $a$. Thus we obtain the MTL formula $\zeta=\delta_{2} \wedge \delta_{3} \wedge \lambda$.

\section{Discussion and Related Work}

Within temporal and real time logics, the notion of counting has attracted considerable interest. Laroussini et al extended untimed LTL with threshold counting constrained until operator. They showed that the expressiveness of LTL is not increased by adding threshold counting but the logic become exponentially more succinct. Hirshfeld and Rabinovich introduced $\mathrm{C}_{(0,1)}$ operator in continuous timed QTL and showed that it added expressive power. They also showed that in continuous time, more general $\mathrm{C}_{\langle l, u\rangle}$ operator can be expressed with just $\mathrm{C}_{(0,1)}$. Building upon this, Hunter showed that MTL with $\mathrm{C}_{(0,1)}$ operator is expressively complete w.r.t. $\mathrm{FO}[+, 1]$. Thus it can also express UT operator which is straightforwardly modelled in $\mathrm{FO}[+, 1]$.

In this paper, we have explored the case of MTL with counting operators over timed words interpreted in pointwise manner. We have shown that both $C_{I}$ and UT operators add expressive power to MTL. Moreover, the two operators are independent in the sense that neither can be expressed in terms of the other and MTL. (We use prefixes $C$ and $\mathrm{T}$ to denote a logic extended with $\mathrm{C}$ and UT operators respectively). It is easy to show

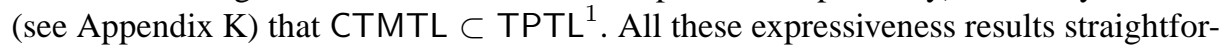
wardly carry over to MTL over infinite timed words. Thus, pointwise semantics exhibits considerable complexity in expressiveness of operators as compared to continuous time semantics where all these logics are equally expressive. While this may arguably be considered a shortcoming of the pointwise models of timed behaviours, the pointwise models have superior decidability properties making them more amenable to algorithmic analysis. MTL already has undecidable satisfiability in continuous time whereas it has decidable satisfiability over finite timed words in pointwise semantics.

In this paper, we have shown that MTL extended with C and UT operators also has decidable satisfiability. The result is proved by giving an equisatisfiable reduction from CTMTL to MTL using the technique of oversampling projections. This technique was introduced earlier [3] and used to show that $\operatorname{MTL}\left[\mathrm{U}_{I}, \mathrm{~S}_{\mathrm{np}}\right]$ with non-punctual past operator is also decidable in pointwise semantics. Current paper marks one more use of the technique of oversampling projections. A closer examination of our reduction from CTMTL to MTL shows that it can be used in presence of any other operator. Also, it does not introduce any punctual use of $\mathrm{U}_{I}$ in reduced formula. The reduced formula is exponentially larger than the original formula (assuming binary encoding of integer constants). All this implies that CTMTL[ $\left.\mathrm{U}_{I}, \mathrm{~S}_{\mathrm{np}}\right]$ is also decidable over finite timed words. Moreover, CTMITL $\left[U_{N S}, S_{N S}\right]$ can be equisatisfiably reduced to MITL $\left[U_{n p}, S_{n p}\right]$ 
and it is decidable with at most 2-EXPSPACE complexity. The exact complexity of satisfiability checking of CTMITL is open although EXPSPACE lowerbound trivially follows from MITL and counting LTL which are syntactic subsets.

In another line of work involving counting and projection, Raskin [12] extended MITL and event clock logic with ability to count by extending these logics with automaton operators and adding second order quantification. The expressiveness was shown to be that of recursive event clock automaton. These logics were able to count over the whole model rather than a particular timed interval. The resultant logic cannot specify constraints like within a time unit $(0,1)$ the number of occurrence of a particular formula is $k$ but can also incorporate mod counting. Thus Raskin's logics and the CTMTL are expressively independent. 


\section{References}

1. Y. Hirshfeld and A. Rabinovich. An expressive temporal logic for real time. In MFCS, pages 492-504, 2006.

2. P. Hunter. When is metric temporal logic expressively complete? In CSL, pages 380-394, 2013.

3. S. N. Krishna K. Madnani and P. K. Pandya. Partially punctual metric temporal logic is decidable. In TIME, pages 174-183, 2014.

4. D. Kini, S. N. Krishna, and P. K. Pandya. On construction of safety signal automata for MITL $[\mathcal{U}, \mathcal{S}]$ using temporal projections. In FORMATS, pages 225-239, 2011.

5. F. Laroussinie, A. Meyer, and E. Petonnet. Counting ltl. In TIME, pages 51-58, 2010.

6. K. Madnani, S. N. Krishna, and P. K. Pandya. Partially punctual metric temporal logic is decidable. In http://arxiv.org/abs/1404.6965, 2014.

7. J. Ouaknine and J. Worrell. On the decidability of metric temporal logic. In LICS, pages 188-197, 2005.

8. F. Chevalier P. Bouyer and N. Markey. On the expressiveness of tptl and mtl. In FST\&TCS, pages 432-443, 2005.

9. P. K. Pandya and S. Shah. On expressive powers of timed logics: Comparing boundedness, non-punctuality, and deterministic freezing. In CONCUR, pages 60-75, 2011.

10. Pavithra Prabhakar and Deepak D'Souza. On the expressiveness of MTL with past operators. In FORMATS, pages 322-336, 2006.

11. A. Rabinovich. Complexity of metric temporal logics with counting and the pnueli modalities. Theor. Comput. Sci., 411(22-24):2331-2342, 2010.

12. Jean Francois Raskin. Logics, Automata and Classical Theories for Deciding Real Time. PhD thesis, Universite de Namur, 1999.

13. H. Straubing. Finite Automata, Formal Logic and Circuit Complexity. Birkhauser, 1994. 


\section{Appendix}

\section{A Motivation}

Example 1. Our first example is motivated from medical devices used in monitoring foetal heart rate. In neo-natal care, the use of external and internal foetal heart rate monitoring devices is well-known. The average foetal heart rate is between 110 and 160 beats per minute, and can vary 5 to 25 beats per minute. An abnormal foetal heart rate ( $<100$ beats per minute or $>180$ beats per minute) may indicate that the foetus is not getting enough oxygen or that there are other problems. Current techniques rely predominantly on the use of electronic foetal monitoring through the use of cardiotocography (CTG). This technique records changes in the foetal heart rate (FHR) (via Doppler ultrasound or direct foetal ECG measurement with a foetal scalp electrode) and their temporal relationship to myometrial activity and uterine contractions. In high risk cases, the electronid foetal monitoring is combined with checking the mother's blood oxygen saturation levels. Normal blood oxygen levels are considered 95-100 percent. These are specialised real-time properties that need to be formally specified in order to model check important safety properties in medical devices. These properties are not only time critical, but also need to measure the number of times an event occurs in a given interval of time, to ensure safety. Let the proposition fhb denote a foetal heart beat, and let the proposition $\mathrm{sp}-$ ok denote normal blood oxygen levels of the mother. The CMTL formula $\square_{[0,60]}$ spo-ok $\wedge C_{[0,60]}^{\geq 110}$ (fhb) $\wedge C_{[0,60]}^{\leq 160}$ (fhb) specifies that in a duration of 60 seconds, the mother's blood oxygen levels are normal, while the foetal heart beats in the range of $[110,160]$.

Example 2. Our second example is motivated from the problem of energy peak reduction in large organisations using HVAC systems. The problem of energy peak demand reduction within a large organization by synchronizing switching decisions of various "heating, ventilation, and air conditioning" (HVAC) systems is one of the most practically relevant ones. The relationship between energy demand peaks and extreme climatic conditions has been studied in the literature; hence, reducing the energy peak demand of HVAC systems can significantly reduce the power peak demand. Nghiem et al. considered the model of an organization divided into various zones, where at any given point of time, the HVAC system of a zone can be switched off or switched on to ensure that the zone stays in a comfortable temperature range. Several scheduling algorithms for the same have been proposed so far in the literature, with the restriction that simultaneously a bounded number of HVAC systems are switched on at any point in time. Also, the number of times a HVAC unit oscillates between the on and off mode should be minimal, while respecting the comfortable temperature range in each zone. Synthesizing the optimal number of HVAC units that have to remain switched on to maintain the comfort level in any zone is an important research problem. We motivate the use of our counting logics to specify the number of times an HVAC unit switches between the on and the off mode. Let hvac ${ }_{1}^{i}$ be a proposition that evaluates to true when a HVAC in zone $i$ has just been switched on, and let hvac $c_{1}^{i}$ be a proposition that evaluates to true when a HVAC in zone $i$ has just been switched off. Let $I$ be the set of zones and 
zone $_{\text {high_temp }}^{i}$ be a proposition which evaluates to true when a zone $i$ is not in its comfortable temperature zone, and let zone $e_{\mathrm{cz}}^{i}$ be a proposition which evaluates to true when a zone $i$ is in its comfortable temperature zone. Let $\psi_{x}=\operatorname{hvac}_{1-x}^{i} \wedge \neg \operatorname{hvac}_{x}^{i} \mathrm{U} \mathrm{hvac}_{x}^{i}$ and let $\eta=\bigvee_{x \in\{0,1\}} \psi_{x}$. The TMTL formula $\bigwedge_{i \in I} \square^{\mathrm{w}}\left(\right.$ zone $_{\text {high_temp }}^{i} \rightarrow \nabla_{\left\{[0, u], \#_{n} \leq n\right\}}\left(\right.$ zone $\left.\left._{\mathrm{cz}}^{i}\right)\right)$ specifies that any zone which is not in the comfortable range should reach the comfort zone in no more than $u$ time units, and while reaching there the number of switches from on to off or off to on of any HVAC in the zone is at most $n$ times. One may also want to control the average number of times the switching happens between on and off. The CMTL formula $\square^{\mathrm{w}}\left[\mathrm{C}_{[0,1]}^{<c} \bigvee_{i \in I} \mathrm{hvac}^{i}\right]$ where $\mathrm{hvac}^{i}=\mathrm{hvac}_{1}^{i} \vee \mathrm{hvac}_{1}^{i} \mathrm{spec}$ ifies that from any event within $[0,1]$ the number of times any HVAC is switched on or off is $<c$. These counting logics can be used to model check the HVAC scheduling algorithms; it is also possible to rewrite these algorithms in the counting logics. Satisfiability checking of these logics can then be used to find the optimal number $k$ of HVAC systems that are required to be on to ensure a comfort temperature range in any zone for a given time interval. Assuming that the environment behaviour and the scheduling algorithm is given in some declarative form, satisfiability checking of the formula $\diamond_{\{[l, u], \# \text { switches }<w\}}\left(\bigwedge_{i \in I} \neg\right.$ zone $_{\text {high_temp }} \rightarrow$ Algo $_{k} \wedge$ environment_parameter $)$ for various values of $k$ and finding the minimal such $k$ tells the optimal number of HVAC units that should remain switched on per zone.

\section{B CTMTL with General Threshold Formulae}

We now generalize the threshold modality used in CTMTL as follows: For $\varphi \in$ CTMTL, and $\sim \in\{<, \leq, \geq,>\}$

$$
\eta:=\# \varphi \sim c|\eta \wedge \eta| \eta \vee \eta \mid \neg \eta
$$

We show in this section that any formula in CTMTL that is written with a complex threshold formula can be rewritten in terms of simple threshold formulae as introduced in Section 2 while introducing CTMTL.

Lemma 8. Let $\varphi \mathrm{U}_{I, \eta} \psi \in \mathrm{CTMTL}$ with $\eta=\eta_{1} \vee \cdots \vee \eta_{n}$. Then $\varphi \mathrm{U}_{I, \eta} \psi$ is equivalent to $\varphi \mathrm{U}_{I, \eta_{1}} \psi \vee \varphi \mathrm{U}_{I, \eta_{2}} \psi \vee \cdots \vee \varphi \mathrm{U}_{I, \eta_{n}} \psi$.

Proof. Let $\eta_{i}=\# \varphi_{i} \sim m_{i}$ for $1 \leq i \leq n$. Given a timed word $\rho$ and a point $i \in$ $\operatorname{dom}(\rho), \rho, i \models \varphi \mathrm{U}_{I, \eta} \psi$ iff there is a point $j>i$ such that $\rho, j \models \psi$, and $\varphi$ evaluates to true at all the in between points $i<k<j$, and there is atleast one formula $\varphi_{i}$ such that the number of points between $i$ and $j$ where $\varphi_{i}$ evaluates to true is $\sim m_{i}$. Hence we obtain $\rho, i \models \varphi \mathrm{U}_{I, \eta_{r}} \psi$, for some $1 \leq r \leq n$. The converse is similar.

A threshold formula $\eta$ is called atomic iff all the threshold formulae $\eta_{j}$ occurring in $\eta$ cannot be written as the conjunction or disjunction of two threshold formulae. Thus, the threshold formula $\eta=\#\left(a \bigcup_{J, \# b=5 \wedge \# c<3}\right) \geq 5$ is not atomic, since it involves a conjunction of two threshold formulae. 
It can be easily seen that every threshold formula $\eta$ is equivalent to some threshold formula $\eta_{1}$ in disjunctive normal form. The formula $\eta_{1}$ in DNF is obtained by recursively replacing all the threshold formulae occurring in $\eta$ in DNF.

For instance, $\eta=[\# b=5] \wedge\left[\#\left(a \bigcup_{I} b\right)<7\right] \vee\left[\#\left(a \bigcup_{\{J, \# d<12 \wedge \# e<6\}} c\right) \geq 2\right]$ can be expressed as $\left[\# b=5 \wedge \#\left(a \mathrm{U}_{I} b\right)<7\right] \vee\left[\#\left[\left(a \bigcup_{\{J, \# d<12\}} c\right) \wedge\left(a \bigcup_{\{J, \# e<6\}} c\right)\right] \geq 2\right] .5$ Without loss of generality, we assume henceforth that every UT modality $\varphi \mathrm{U}_{I, \eta} \psi$ we encounter in CTMTL formulae has $\eta$ in DNF. The following two lemmas on monotonicity of counting with respect to time are easy to follow.

Lemma 9. Let $\delta=\# \delta_{1}<n_{1} \wedge \ldots \# \delta_{m}<n_{m}$ be a threshold formula. Let $\rho$ be a timed word and let $x<y$ be two points in $\operatorname{dom}(\rho)$. Assume that $\left|\rho[x, y]\left(\delta_{i}\right)\right|<n_{i}$ for all $1 \leq i \leq m$. Then for any $y^{\prime} \in \operatorname{dom}(\rho)$, with $x<y^{\prime}<y$, we have $\left|\rho\left[x, y^{\prime}\right]\left(\delta_{i}\right)\right|<n_{i}$ for all $1 \leq i \leq m$.

Lemma 10. Let $\delta=\# \delta_{1} \geq n_{1} \wedge \ldots \# \delta_{m} \geq n_{m}$ be a threshold formula. Let $\rho$ be a timed word and let $x<y$ be two points in $\operatorname{dom}(\rho)$. Assume that $\left|\rho[x, y]\left(\delta_{i}\right)\right| \geq n_{i}$ for all $1 \leq i \leq m$. Then for any $y^{\prime} \in \operatorname{dom}(\rho)$, with $y^{\prime}>y$, we have $\left|\rho\left[x, y^{\prime}\right]\left(\delta_{i}\right)\right| \geq n_{i}$ for all $1 \leq i \leq m$.

Lemma 11. Let $\varphi \mathrm{U}_{I, \eta} \psi \in \mathrm{CTMTL}$. Let $\eta=\alpha \wedge \beta$, where $\alpha=\# \alpha_{1} \geq n_{1} \wedge$ $\ldots \# \alpha_{m} \geq n_{m}$ and $\beta=\# \beta_{1}<k_{1} \wedge \ldots \# \beta_{p}<k_{p}$. Then $\varphi \mathrm{U}_{I, \eta} \psi$ is equivalent to $\varphi \mathrm{U}_{[0, \infty), \eta} \psi \wedge \bigwedge_{i=1}^{m} \varphi \mathrm{U}_{I, \# \alpha_{i} \geq n_{i}} \psi \wedge \bigwedge_{i=1}^{p} \varphi \mathrm{U}_{I, \# \beta_{i}<k_{i}} \psi$.

Proof. Let $\eta=\alpha \wedge \beta$, where $\alpha$ is the conjunction of all the threshold formulae with comparison operator $\geq$ occurring in $\eta$ and $\beta$ is the conjunction of all the threshold formulae with comparison operator $<$ occurring in $\eta$. Let $\alpha=\#_{\alpha_{1}} \geq n_{1} \wedge \ldots \#_{\alpha_{m}} \geq$ $n_{m}$ and $\beta=\#_{\beta_{1}}<k_{1} \wedge \ldots \#_{\beta_{p}}<k_{p}$. Let $\rho$ be a timed word and let $i \in \operatorname{dom}(\rho)$. $\rho, i \models \varphi \mathrm{U}_{I, \eta} \psi$ iff there is a point $j>i$ with $t_{j} \in t_{i}+I$, and the number of points in between $i$ and $j$ where $\alpha_{r}$ evaluates to true is $\geq n_{r}$ for $1 \leq r \leq m$ and the number of points between $i$ and $j$ where $\beta_{l}$ evaluates to true is $<k_{l}$ for $1 \leq l \leq p$.

It is easy to see that $\varphi \mathrm{U}_{I, \eta} \psi \rightarrow \varphi \mathrm{U}_{[0, \infty), \eta} \psi \wedge \varphi \mathrm{U}_{I, \alpha} \psi \wedge \varphi \mathrm{U}_{I, \beta} \psi$.

Conversely, assume that $\rho, i \models \varphi \mathrm{U}_{\eta} \psi \wedge \varphi \mathrm{U}_{I, \alpha} \psi \wedge \varphi \mathrm{U}_{I, \beta} \psi$.

1. Since $\rho, i \models \varphi \mathrm{U}_{\eta} \psi$, there is a point $j_{1}>i$ such that $\psi$ evaluates to true at $j_{1}$, $\varphi$ evaluates to true at all points between $i$ and $j_{1}$, and $\left|\rho\left[i, j_{1}\right]\left(\alpha_{r}\right)\right| \geq n_{r}$ for all $1 \leq r \leq m$ and $\left|\rho\left[i, j_{1}\right]\left(\beta_{l}\right)\right|<k_{l}$ for all $1 \leq l \leq p$.

2. Since $\rho, i \models \varphi \mathrm{U}_{I, \alpha} \psi$, there is a point $j_{2}>i$ such that $\psi$ evaluates to true at $j_{2}, \varphi$ evaluates to true at all points between $i$ and $j_{2}, t_{j_{2}}-t_{i} \in I$, and $\left|\rho\left[i, j_{2}\right]\left(\alpha_{r}\right)\right| \geq n_{r}$ for all $1 \leq r \leq m$.

3. Since $\rho, i \models \varphi \mathrm{U}_{I, \beta} \psi$, there is a point $j_{3}>i$ such that $\psi$ evaluates to true at $j_{3}, \varphi$ evaluates to true at all points between $i$ and $j_{3}, t_{j_{3}}-t_{i} \in I$, and $\left|\rho\left[i, j_{3}\right]\left(\beta_{l}\right)\right|<k_{l}$ for all $1 \leq l \leq p$.

Assume $j_{2} \leq j_{3}$. We will check whether $j_{2}>i$ is the point which satisfies all the conditions required with respect to $I, \alpha$ and $\beta$. Since the number of points between $i$ and $j_{3}$ where $\beta_{l}$ evaluates to true is $<k_{l}$ for all $1 \leq l \leq p$, and $j_{2} \leq j_{3}$, by monotonicity

\footnotetext{
${ }^{5}$ see the proof in the next paragraph, Lemma 11
} 
of time (Lemma9), the number of points between $i$ and $j_{2}$ where $\beta_{l}$ evaluates to true is $<k_{l}$ for all $1 \leq l \leq p$. Also, we know that the number of points between $i$ and $j_{2}$ where $\alpha_{r}$ evaluates to true is $\geq n_{r}$ for all $1 \leq r \leq m$. Then indeed we have $\rho, i \models \varphi \mathrm{U}_{I, \eta} \psi$.

Consider the case when $j_{3}<j_{2}$. Assume that there is some $\beta_{l}$ such that $\left|\rho\left[i, j_{2}\right]\left(\beta_{l}\right)\right| \geq$ $k_{l}$. Since we know that $j_{1}>i$ is a point such that $\left|\rho\left[i, j_{1}\right]\left(\beta_{l}\right)\right|<k_{l}$ for all $1 \leq l \leq p$, it must be that $j_{1}<j_{2}$. If there is some $\alpha_{h}$ such that $\left|\rho\left[i, j_{3}\right]\left(\alpha_{h}\right)\right|<n_{h}$, then again by monotonicity of time (Lemma 10 ), we know that $j_{1} \geq j_{3}$. So we have $j_{3} \leq j_{1}<j_{2}$. Hence, $t_{j_{1}} \in t_{i}+I$ since $t_{j_{3}}-t_{i} \in I$ and $t_{j_{2}}-t_{i} \in I$. Thus, we have a point $j_{1}>i$ such that $t_{j_{1}}-t_{i} \in I$, satisfying all the conditions. Hence, $\rho, i \models \varphi \mathrm{U}_{I, \eta} \psi$.

Now we show that $\rho, i \models \varphi \mathrm{U}_{I, \alpha} \psi$ iff $\rho, i \models \bigwedge_{i=1}^{m} \varphi \mathrm{U}_{I, \# \alpha_{i} \geq n_{i}} \psi$. The equivalence of $\varphi \mathrm{U}_{I, \beta} \psi$ and $\bigwedge_{i=1}^{p} \varphi \mathrm{U}_{I, \# \beta_{i}<k_{i}} \psi$ is similar.

Assume $\rho, i \models \varphi \mathrm{U}_{I, \alpha} \psi$. Then it is easy to see that $\rho, i \models \bigwedge_{i=1}^{m} \varphi \mathrm{U}_{I, \# \alpha_{i} \geq n_{i}} \psi$. Conversely, assume that $\rho, i \models \bigwedge_{i=1}^{m} \varphi \mathrm{U}_{I, \#_{\alpha_{i}} \geq n_{i}} \psi$. Then there are points $j_{1}, \ldots, j_{m}>$ $i$ such that $t_{j_{i}}-t_{i} \in I, \psi$ evaluates to true at $j_{i}, \varphi$ evaluates to true at all points between $i$ and $j_{i}$, and $\left|\rho\left[i, j_{i}\right]\left(\alpha_{i}\right)\right| \geq n_{i}$ for all $1 \leq i \leq m$. Let $j_{k}$ be the point among $j_{1}, \ldots, j_{m}$ that is farthest from $i$. Then clearly, by monotonicity of time (Lemma 10), $\left|\rho\left[i, j_{k}\right]\left(\alpha_{i}\right)\right| \geq n_{i}$ for all $1 \leq i \leq m$. Hence, $j_{k}>i$ is the point which satisfies all the conditions required of $\varphi \mathrm{U}_{I, \alpha} \psi$, and hence, $\rho, i \models \varphi \mathrm{U}_{I, \alpha} \psi$.

\section{Recalling MTL games from [9]}

An $r$-round $I_{\nu} \mathrm{MTL}$ game is played between two players (Spoiler and Duplicator) on a pair of timed words $\left(\rho_{1}, \rho_{2}\right)$, where $I_{\nu}$ is the set of intervals allowed in the game. A configuration of the game is a pair of points $i_{p}, j_{p}$ where $i_{p} \in \operatorname{dom}\left(\rho_{1}\right)$ and $j_{p} \in$ $\operatorname{dom}\left(\rho_{2}\right)$. A configuration is called partially isomorphic, denoted $i \operatorname{sop}\left(i_{p}, j_{p}\right)$ iff $\sigma_{i_{p}}=$ $\sigma_{j_{p}}$. The starting configuration is $\left(i_{1}, j_{1}\right)$. Either Spoiler or Duplicator eventually wins the game. A 0 -round game is won by the Duplicator iff $i s o p\left(i_{1}, j_{1}\right)$. The $r$ round game is played by first playing one round from the starting position. Either the Spoiler wins the round, and the game is terminated or the Duplicator wins the round, and now the second round is played from this new configuration and so on. The Duplicator wins the game only if he wins all the rounds. The following are the rules of the game in any round. Assume that the configuration at the start of the $p$ th round is $\left(i_{p}, j_{p}\right)$.

- If $i \operatorname{sop}\left(i_{p}, j_{p}\right)$ is not true, then Spoiler wins the game, and the game is terminated. Otherwise, the game continues as follows:

- The Spoiler chooses one of the words by choosing $\rho_{x}, x \in\{1,2\}$. Duplicator has to play on the other word $\rho_{y}, x \neq y$. Then Spoiler chooses the $\mathrm{U}_{I}$ move, along with the interval $I \in I_{\nu}$ (such that the end points of the intervals are non-negative integers). Given the current configuration as $\left(i_{p}, j_{p}\right)$, the rest of the $\mathrm{U}_{I}$ round is played as follows:

- Spoiler chooses a position $i_{p}^{\prime} \in \operatorname{dom}\left(\rho_{x}\right)$ such that $i_{p}<i_{p}^{\prime}$ and $\left(t_{i_{p}^{\prime}}-t_{i_{p}}\right) \in I$.

- The Duplicator responds to the $\mathrm{U}_{I}$ move by choosing $j_{p}^{\prime} \in \operatorname{dom}\left(\rho_{y}\right)$ in the other word such that $j_{p}<j_{p}^{\prime}$ and $\left(t_{j_{p}^{\prime}}-t_{j_{p}}\right) \in I$. If the Duplicator cannot find such a position, the Spoiler wins the round and the game. Otherwise, the game continues and Spoiler chooses one of the following options. 
- $\diamond$ Part: The round ends with the configuration $\left(i_{p}^{\prime}, j_{p}^{\prime}\right)$.

- U Part: Spoiler chooses a position $j_{p}^{\prime \prime}$ in $\rho_{y}$ such that $j_{p}<j_{p}^{\prime \prime}<j_{p}^{\prime}$. The Duplicator responds by choosing a position $i_{p}^{\prime \prime}$ in $\rho_{x}$ such that $i_{p}<i_{p}^{\prime \prime}<i_{p}^{\prime}$. The round ends with the configuration $\left(i_{p}^{\prime \prime}, j_{p}^{\prime \prime}\right)$. If the Duplicator cannot choose an $i_{p}^{\prime \prime}$, the game ends and the Spoiler wins.

- Game equivalence: $\left(\rho_{1}, i_{1}\right) \approx_{r, I_{\nu}}\left(\rho_{2}, j_{1}\right)$ iff for every $r$-round MTL game over the words $\rho_{1}, \rho_{2}$ starting from the configuration $\left(i_{1}, j_{1}\right)$, the Duplicator always has a winning strategy.

- Formula equivalence: $\left(\rho_{1}, i_{1}\right) \equiv_{r, I_{\nu}}^{\mathrm{MTL}}\left(\rho_{2}, j_{1}\right)$ iff for every MTL formula $\phi$ of modal depth $\leq r, \rho_{1}, i_{1} \models \phi \Longleftrightarrow \rho_{2}, j_{1} \models \phi$

Theorem 5. $\left(\rho_{1}, i_{1}\right) \approx_{r, I_{\nu}}\left(\rho_{2}, j_{1}\right)$ iff $\left(\rho_{1}, i_{1}\right) \equiv_{r, I_{\nu}}^{\mathrm{MTL}}\left(\rho_{2}, j_{1}\right)[9]$.

\section{Proof of Theorem 4}

We prove the result for theorem 4 in this section using structural induction on the number $r$ of rounds. We first observe that in the base case $r=0$, the theorem holds: If $\left(\rho_{1}, i_{1}\right) \approx_{0, k, I_{\nu}}\left(\rho_{2}, j_{1}\right)$, Duplicator wins the zero round game. This is possible iff $i \operatorname{sop}\left(i_{1}, j_{1}\right)$. It is then clear that both words satisfy the same formulae of depth 0 . The converse is similar.

Assume the theorem holds for $r=n$ rounds. We will prove the theorem for $n+1$ rounds.

1. Assume $\left(\rho_{1}, i_{1}\right) \approx_{n+1, k, I_{\nu}}\left(\rho_{2}, j_{1}\right)$. Let us consider $\varphi=\psi \mathrm{U}_{I, \#_{\delta} \geq w} \phi$. Assume further that $\rho_{1}, i_{1} \models \varphi$. We need to prove that $\rho_{2}, j_{1} \models \varphi$.

(a) Let us first consider the case when Spoiler initiates a $\nabla_{I}$ move on $\rho_{1}$. Let $i_{1}^{\prime}$ be the point chosen. Duplicator has to mimic the move by choosing a point $j_{1}^{\prime}$. If Spoiler ends the round at this point, then by assumption, we know that Duplicator wins from $\left(i_{2}, j_{2}\right)=\left(i_{1}^{\prime}, j_{1}^{\prime}\right)$ in an $n$ round game. By induction hypothesis, we know that $i_{2}$ and $j_{2}$ satisfy the same set of formulae with depth $\leq n$. Thus $\rho_{2}, j_{2} \models \phi$.

(b) Now consider the case that Spoiler plays a full until round from $\left(i_{1}, j_{1}\right)$. Then he chooses a point $j_{1}^{\prime \prime}\left(j_{1}<j_{1}^{\prime \prime}<j_{1}^{\prime}\right)$ in Duplicator's word. By assumption, duplicator will be able to choose a point $i_{1}^{\prime \prime}\left(i_{1}<i_{1}^{\prime \prime}<i_{1}^{\prime}\right)$ such that he wins the game from $\left(i_{2}, j_{2}\right)=\left(i_{1}^{\prime \prime}, j_{1}^{\prime \prime}\right)$ in the next $n$ rounds. By induction hypothesis, all points between $i_{1}, i_{1}^{\prime}$ as well as between $j_{1}, j_{1}^{\prime}$ satisfy the same set of formulae of depth $\leq n$. We know that the depth of $\psi$ is $\leq n$, and all points between $i_{1}$ and $i_{1}^{\prime}$ satisfy $\psi$. Thus all the points strictly between $j_{1}$ and $j_{1}^{\prime}$ satisfy $\psi$. Hence, $\rho_{2}, j_{2} \models \psi$.

(c) The third choice of the Spoiler is to invoke the UT move. Spoiler keeps $w<k$ pebbles between $i_{1}$ and $i_{1}^{\prime}$. Let $I_{1}$ be the set of pebbled points in Spoiler's word. In response, Duplicator also keeps $w$ pebbles in his word between $j_{1}$ and $j_{1}^{\prime}$. Let $I_{2}$ be the set of pebbled positions in Duplicator's word. For any choice of a pebbled point $j_{2} \in I_{2}$, Duplicator picks some point $i_{2} \in I_{1}$. By assumption, Duplicator wins an $n$ round game from this configuration. Hence, 
all the pebbled positions in both words satisfy the same set of formulae of depth $\leq n$, and in particular $\delta$. Hence, $\rho_{2}, j_{2} \models \delta$.

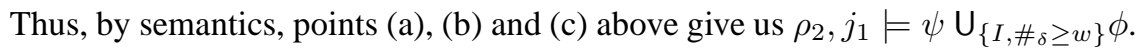

2. We now consider the case when the outer most connective is a $C$ modality. Let $\varphi=\mathrm{C}_{\langle l, u\rangle}^{\geq w} \delta$. Assume that $\rho_{1}, i_{1} \models \varphi$. We need to prove that $\rho_{2}, j_{1} \models \varphi$. Spoiler selects $w \leq k$ points with timestamps in $\left\langle t_{i_{1}}+l, t_{i_{1}}+u\right\rangle$ that satisfies $\delta$ and keep his pebbles. Let $I_{1}$ be the set of points pebbled by Spoiler. In response, the Duplicator chooses $w$ points with timestamps $\left\langle t_{j_{1}}+l, t_{j_{1}}+u\right\rangle$. Let $I_{2}$ be the set of points pebbled by Duplicator. Spoiler chooses a point from $I_{2}$; the duplicator responds with a point in $I_{1}$. By assumption, for any point $e_{2} \in I_{2}$ chosen by Spoiler, the Duplicator can pick a point in $e_{1} \in I_{1}$ such that from $\left(e_{1}, e_{2}\right)$, Duplicator wins in the next $n$ rounds. By induction hypothesis, $\forall e_{2} \in I_{2}, \exists e_{1} \in I_{1},\left(\rho_{1}, e_{1}\right) \equiv_{n, k}$ $\left(\rho_{2}, e_{2}\right)$. Note that all the points in $I_{1}$ satisfy $\delta$. Since $\delta$ has depth $n$, all the points in $I_{2}$ also satisfy $\delta$. Thus $\rho_{2}, j_{1}$ also satisfies $\mathrm{C}_{\langle l, u\rangle}^{\geq w} \delta$. Hence, $\rho_{2}, j_{1} \models \varphi$.

We will now prove the contrapositive. If $\left(\rho_{1}, i_{1}\right) \approx_{0, k, I_{\nu}}\left(\rho_{2}, j_{1}\right)$, then $\neg i s o p\left(i_{1}, j_{1}\right)$. Then clearly, there is a depth 0 formula that distinguishes $\rho_{1}, \rho_{2}$. Let us assume the result for $r=n$ and let $\left(\rho_{1}, i_{1}\right) \nsim_{n+1, k, I_{\nu}}\left(\rho_{2}, j_{1}\right)$. We construct a formula of depth $n+1$ that separates $\rho_{1}, i_{1}$ and $\rho_{2}, j_{1}$. Given $\rho_{1}, \rho_{2}$ of finite length say $n, m$ respectively, the choice of intervals $I_{\nu}=\{\langle i, j\rangle \mid 0 \leq i \leq \max (n, m), 0 \leq j \leq \max (n, m)$ or $j=\infty$ and $i \leq j\}$.

1. Assume without loss of generality that Spoiler chooses $\rho_{1}, i_{1}$ to start with and plays a $\nabla_{I}$ by choosing $i_{1}^{\prime}>i_{1}$. Duplicator chooses a point $j_{1}^{\prime}>j_{1}$ in $\rho_{2}$. If Spoiler wins from $\left(i_{1}^{\prime}, j_{1}^{\prime}\right)=\left(i_{2}, j_{2}\right)$, then by induction hypothesis, there is an $n$ depth formula which evaluates to true at $\rho_{1}, i_{1}^{\prime}$ but not at $\rho_{2}, j_{1}^{\prime}$. Let $Q_{x}$ be the conjunction of all depth $n$ formulae that evaluate to true at $\rho_{1}, x$. For a given $n, k$ and permitted intervals $I_{\nu}$, this conjunction is bounded and finite : Thus, if Spoiler wins after the $\nabla_{I}$ round, the formula $\nabla_{I}\left(Q_{j_{1}^{\prime}}\right)$ of depth $n+1$ distinguishes the words.

2. Suppose that Spoiler has to play an $\mathrm{U}_{I}$ round to win the $n+1$ round game. Then Spoiler picks a point $j_{1}^{\prime \prime}$ between $j_{1}$ and $j_{1}^{\prime}$ in $\rho_{2}$. For any point $i_{1}^{\prime \prime}$ between $i_{1}$ and $i_{1}^{\prime}$ picked by the Duplicator, the $n$ round game is won by Spoiler. Thus, there exists a point $j_{1}<x<j_{1}^{\prime}$ and some formula of depth $n$ which distinguishes $x$ from all the points between $i_{1}$ and $i_{1}^{\prime}$. Consider the formula $P=\underset{y \in\left\{i_{1}, \ldots i_{1}^{\prime}\right\}}{\bigvee} Q_{y}$. The size of $P$ is bounded since the size of each $Q_{y}$ is bounded, and the number of disjuncts is finite. Hence, there is a point $j_{1}^{\prime \prime}\left(j_{1}<j_{1}^{\prime \prime}<j_{1}^{\prime}\right)$ such that $\rho_{2}, j_{1}^{\prime \prime} \not \models P$. Thus the formula distinguishing $\rho_{1}, \rho_{2}$ is $P \mathrm{U}_{I} Q_{i_{1}^{\prime}}$.

3. Suppose that Spoiler has to play the UT round to win the game. Assume Spoiler chose the word $\rho_{1}$ and places his $w \leq k$ pebbles at a set of points $I_{1}$ between $i_{1}$ and $i_{1}^{\prime}$. In response, Duplicator keeps his $w \leq k$ pebbles at a set of points $I_{2}$ between $j_{1}$ and $j_{1}^{\prime}$. Spoiler picks a point $j_{1}^{\prime \prime} \in I_{2}$, to which Duplicator replies by picking $i_{1}^{\prime \prime} \in I_{1}$. Since Spoiler wins by assumption, there is a formula of depth $\leq n$ that distinguishes $j_{1}^{\prime \prime}$ from all the points in $I_{1}$. Now consider the formula $P_{I_{1}}=\bigvee_{i \in I_{1}} Q_{i}$, where $Q_{i}$ be the conjunction of all depth $n$ formulae that evaluate to true at $\rho_{1}, i$. For a given $n, k$ and permitted intervals $I_{\nu}$, there are a bounded number of $n$ depth 
formulae; hence the number of different formulae $P_{I_{1}}$ is bounded. Since Spoiler wins the game in the next $n$ rounds, $P_{I_{1}}$ is true for at least $w$ number of times between $i_{1}$ and $i_{1}^{\prime}$ since it evaluates to true at all points between $i_{1}$ and $i_{1}^{\prime}$. However, the number of times $P_{I_{1}}$ evaluates to true between $j_{1}$ and $j_{1}^{\prime}$ is $<w$, since it does not evaluate to true at $j_{1}^{\prime \prime}$. Hence, $\rho_{2}, j_{1} \not \models P \mathrm{U}_{I, \# P_{I_{1}} \geq w} Q_{i_{1}^{\prime}}$ where $P=\underset{y \in\left\{i_{1}, \ldots i_{1}^{\prime}\right\}}{\bigvee} Q_{y}$ is as defined above.

Similarly, if Spoiler had pebbled the points $I_{2}$ between $j_{1}$ and $j_{1}^{\prime}$ in the counting part, then Duplicator pebbles the set $I_{1}$ of points between $i_{1}$ and $i_{1}^{\prime}$. Then $P_{I_{2}}=\bigvee_{i \in I_{2}} Q_{i}$ evaluates to true atleast $w$ times between $j_{1}$ and $j_{1}^{\prime}$, but there is some point $i_{1}^{\prime \prime} \in I_{1}$ chosen by Spoiler where $P_{I_{2}}$ is false. Then the number of times $P_{I_{2}}$ evaluates to true is $<w$ between $i_{1}$ and $i_{1}^{\prime}$. In this case, $\rho_{2}, j_{1} \not \models P \mathrm{U}_{I, \# P_{I_{2}}<w} Q_{i_{1}^{\prime}}$. 4. Suppose now that Spoiler has to play a $C$ move to win the game. Assume without loss of generality that Spoiler chooses to play from $\rho_{1}$. Let $Q_{x}$ be the conjunction of all the $n$ depth formulae having $k$ as the maximum counting constant in the C, UT modalities that evaluate to true at a point $x$. Given that $n, k$ and the possible intervals $I_{\nu}$ are finite, the number of formulae $Q_{x}$ is bounded. Let us consider the case that Spoiler's first move is a $\mathrm{C}_{\langle l, u\rangle}^{\geq k}$ move. Spoiler pebbles the set $I_{1}$ of $k$ points in $\left\langle t_{i_{1}}+l, t_{i_{1}}+u\right\rangle$. In response, Duplicator pebbles the set $I_{2}$ of $k$ points in $\left\langle t_{j_{1}}+l, t_{j_{1}}+u\right\rangle$. Spoiler picks a point $e_{2} \in I_{2}$, and Duplicator replies by choosing $e_{1} \in I_{1}$. By assumption, Duplicator loses an $n$ round game from $\left(e_{1}, e_{2}\right)$. Hence, by induction, there is a formula $\varphi$ of depth $n$ which will evaluate to false at $e_{2}$. Consider the formula $Q=\bigvee_{x \in I_{1}} Q_{x} . Q$ is a formula of depth $n$ having $k$ as the maximum counting constant in its counting modalities since each $Q_{x}$ is one such. Clearly, $Q$ evaluates to true at all $k$ points of $I_{1}$; however, the number of points where $Q$ evaluates to true is $<k$ in $I_{2}$. Hence, $\rho_{1}, i_{1} \models \mathrm{C}_{\langle l, u\rangle}^{\geq k} Q$, while $\rho_{2}, j_{1} \models \mathrm{C}_{\langle l, u\rangle}^{<k} Q$. The formula $\mathrm{C}_{\langle l, u\rangle}^{\geq k} Q$ has depth $n+1$ with max constant $k$ in its counting modalities and distinguishes the two words.

Hence, we can show that formula equivalence holds iff Duplicator wins in the associated game.

\section{E Details of Situation 2 in Proposition 1}

Situation 2: Starting from $\left(i_{1}, j_{1}\right)$ with time stamps $(0,0)$, if the Spoiler chooses a $\mathrm{U}_{(0,1) \#_{a} \sim c}$ move and lands up at some point between $x_{1}$ and $y_{1}$, Duplicator will play copy-cat and achieve an identical configuration. Consider the case when Spoiler lands up at $y_{1} 6$. In response, Duplicator moves to $y_{1}^{\prime}$. From configuration $\left(i_{2}, j_{2}\right)$ with time stamps $\left(y_{1}, y_{1}^{\prime}\right)$, consider the case when Spoiler initiates a $\mathbf{U}_{(1,2) \#_{a} \sim c}$ and moves to $z_{2}=1.8+\epsilon<2$. In response, Duplicator moves to the point $z_{2}^{\prime}=2.1>2$. A pebble is kept at the inbetween positions $x_{2}, x_{2}^{\prime}$ respectively. If Spoiler chooses to pick the pebble in Duplicator's word, then we obtain the configuration $\left(i_{3}, j_{3}\right)$ with time

\footnotetext{
${ }^{6}$ The argument when Spoiler lands up at $x_{1}$ or a point in between $x_{1}, y_{1}$ is exactly the same
} 


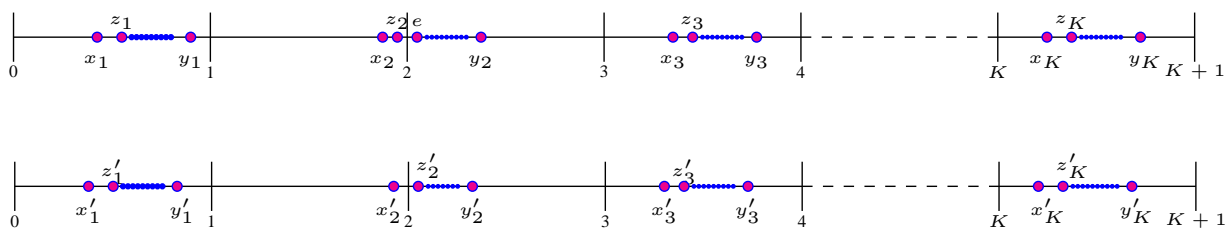

Fig. 4. Words showing CMTL $-T M T L \neq \emptyset$

stamps $\left(x_{2}, x_{2}^{\prime}\right)$. If Spoiler does not get into the counting part/until part, the configuration obtained has time stamps $\left(z_{2}, z_{2}^{\prime}\right)$, with the lag of one segment $\left(\operatorname{seg}\left(i_{3}\right)=1, \operatorname{seg}\left(j_{3}\right)=2\right)$.

- Assume we have the configuration $\left(i_{3}, j_{3}\right)$ with time stamps $\left(x_{2}, x_{2}^{\prime}\right)$. We know that $y_{j}-x_{2}, y_{j}^{\prime}-x_{2}^{\prime}, z_{j}^{\prime}-x_{2}^{\prime}, z_{j}-x_{2} \in(j-1, j)$ and $x_{j}^{\prime}-x_{2}^{\prime}, x_{j}-x_{2} \in(j-2, j-1)$ for $j \geq 3$, and $y_{2}^{\prime}-x_{2}^{\prime}, y_{2}-x_{2}, z_{2}-x_{2}, z_{2}^{\prime}-x_{2}^{\prime} \in(0,1)$. Thus, from $\left(x_{2}, x_{2}^{\prime}\right)$, the possible configuration obtained is $\left(i_{3}^{\prime}, j_{3}^{\prime}\right)=\left(k_{j}, k_{j}^{\prime}\right)$ with $k \in\{x, y, z\}$ and $j \geq 3$ or $\left(z_{2}, z_{2}^{\prime}\right)$ or $\left(y_{2}, y_{2}^{\prime}\right)$. In the case of $\left(z_{2}, z_{2}^{\prime}\right)$, there are no inbetween positions for pebbling. In all the other cases, as long as Spoiler does not keep a pebble on $z_{2}$, we will either obtain $\left(i_{4}, j_{4}\right)=\left(k_{j}, k_{j}^{\prime}\right)$ with $k \in\{x, y, z\}$ and $j \geq 3$ or $\left(y_{2}, y_{2}^{\prime}\right)$. If Spoiler keeps just one pebble, and that too on $z_{2}$, then Duplicator will keep his only pebble at $z_{2}^{\prime}$ obtaining $\left(i_{4}, j_{4}\right)=\left(z_{2}, z_{2}^{\prime}\right)$. In all the cases other than obtaining $\left(i_{4}, j_{4}\right)$ with time stamps $\left(z_{2}, z_{2}^{\prime}\right)$, there is no segment lag. In fact, all these cases give an identical configuration with same time stamps, from where Duplicator can easily win. Lets hence look at the case of $\left(z_{2}, z_{2}^{\prime}\right)$.

- Consider the configuration $\left(i_{3}, j_{3}\right)$ with time stamps $\left(z_{2}, z_{2}^{\prime}\right)$. In this case, there is a lag of one segment.

(a) If Spoiler chooses to move from $z_{2}^{\prime}$ to $x_{3}^{\prime}$, then Duplicator can move to $x_{3}$ from $z_{2}$, since $x_{3}-z_{2}, x_{3}^{\prime}-z_{2}^{\prime} \in(1,2)$ and obtain a configuration $\left(i_{3}^{\prime}, j_{3}^{\prime}\right)$ with same time stamps $\left(x_{3}, x_{3}^{\prime}\right)$. If Spoiler does not pebble the points in between, we obtain an identical configuration with time stamps $\left(x_{3}, x_{3}^{\prime}\right)$, from where it is easy to see that Duplicator wins. If Spoiler pebbles points between $z_{2}$ and $x_{3}$, the interesting situation is when he pebbles only $e$; in this case, Duplicator's best choice is to pebble the point (say $e^{\prime}$ ) right after $z_{2}^{\prime}$ since $e^{\prime}-e=\kappa$. The configuration with time stamps $\left(e, e^{\prime}\right)$ is as good as an identical configuration.

(b) Lets see the case when Spoiler moves to $z_{3}^{\prime}$ or $y_{3}^{\prime}$ from $z_{2}^{\prime}$. Then Duplicator's best choice is to move to $x_{3}$ from $z_{2}$, since he cannot move to $z_{3}, y_{3}$ ( $z_{3}^{\prime}-z_{2}^{\prime} \in(1,2), y_{3}^{\prime}-z_{2}^{\prime} \in(1,2)$, but $\left.z_{3}-z_{2}, y_{3}-z_{2} \in(2,3)\right)$. This gives the configuration $\left(i_{3}^{\prime}, j_{3}^{\prime}\right)$ with time stamps $\left(x_{3}, y_{3}^{\prime}\right)$ or $\left(x_{3}, z_{3}^{\prime}\right)$, with no lag in the segments of $\rho_{1}, \rho_{2}$. If Spoiler pebbles the positions inbetween $z_{2}^{\prime}$ and $y_{3}^{\prime}$ (or $z_{3}^{\prime}$ ), then Duplicator places his pebbles among the bunch of points between $e$ and $y_{2}$. The resultant configuration is $\left(i_{4}, j_{4}\right)$ with the following interesting possibilties: 
(c) $e<t_{i_{4}}<y_{2}$ and $z_{3}^{\prime}<t_{j_{4}}<y_{3}^{\prime}$. From $\left(i_{4}, j_{4}\right)$, if Spoiler moves to any point $k_{j}$ (or $k_{j+1}^{\prime}$ ) for $k \in\{x, y, z\}$ and $j \geq 3$, Duplicator can move into $k_{j+1}^{\prime}\left(\right.$ or $k_{j}$ ) since for any interval $I, k_{j}-t_{i_{4}} \in I$ iff $k_{j+1}^{\prime}-t_{j_{4}} \in I$. This results in future configurations of the kind having time stamps $\left(k_{j}, k_{j+1}^{\prime}\right)$ for $j \geq 3, k \in\{x, y, z\}$, and a segment lag of 1 .

(d) $e<t_{i_{4}}<y_{2}$ and $t_{j_{4}}=x_{3}^{\prime}$. From $\left(i_{4}, j_{4}\right)$, the reachable configurations $\left(i_{4}^{\prime}, j_{4}^{\prime}\right)$ are those where $e<t_{i_{4}^{\prime}}<y_{2}, t_{j_{4}^{\prime}}=z_{3}^{\prime}$, when both players move to the next point $\left(i_{4}^{\prime}=i_{4}+1, j_{4}^{\prime}=j_{4}+1\right)$ or $e<t_{i_{4}^{\prime}}<y_{2}$, $z_{3}^{\prime}<t_{j_{4}^{\prime}}<y_{3}^{\prime}$ (case above) or with time stamps $\left(y_{2}, y_{3}^{\prime}\right)$, or $\left(k_{j}, k_{j+1}^{\prime}\right)$ for $j \geq 3, k \in\{x, y, z\}$. All these result in future configurations of the kind having time stamps $\left(k_{j}, k_{j+1}^{\prime}\right)$ for $j \geq 3, k \in\{x, y, z\}$, and a segment lag of 1 .

(e) $e<t_{i_{4}}<y_{2}$ and $z_{2}^{\prime}<t_{j_{4}}<x_{3}^{\prime}$. This is like an identical configuration, and from here, Duplicator can stay in the same segment as Spoiler in all future moves, obtaining almost identical configurations.

\section{F Proof of Lemma 2}

\section{$\mathrm{MTL} \subseteq \mathrm{C}^{(0,1)} \mathrm{MTL}$}

The containment of MTL in $C^{(0,1)}$ MTL is clear since $C^{(0,1)}$ MTL has all the modalities of MTL. We show strict containment by considering the formula $\varphi=\mathrm{C}_{(0,1)}^{=2} a \in \mathrm{C}^{(0,1)} \mathrm{MTL}$. We show that for any choice $n$ of rounds, we can find two timed words $\rho_{1}, \rho_{2}$ such that $\rho_{1} \models \varphi, \rho_{2} \not \models \varphi$, but $\rho_{1} \equiv{ }_{n}^{\mathrm{MTL}} \rho_{2}$.

Consider the timed words $\rho_{1}=(a, 0)(a, 0.5)(a, 0.6) W$ and $\rho_{2}=(a, 0)(a, 0.5) W$ where $W$ is $(a, 1.1)(a, 1.1+\delta)(a, 1.1+2 \delta) \ldots(a, 1.1+n \delta)$, where $\delta<<\frac{1}{n}$ is some small constant such that $1.1+n \delta<1.2$. Clearly, $\rho_{1} \models \varphi$ and $\rho_{2} \not \models \varphi$. Since the words are identical from time 1.1 onwards, the interesting parts of the game are in the interval $(0,1)$.

Proposition 2. In any round $p$ of the MTL game, Duplicator can always ensure an identical configuration $\left(i_{p}, j_{p}\right)\left(i_{p}=j_{p}\right)$ or ensure that $\left|i_{p}-j_{p}\right| \leq 1$. If $i_{p}-j_{p}=1$ and $i_{p} \geq 3$, then for all $q>p$, Duplicator can ensure that $0 \leq i_{q}-j_{q} \leq 1$. Further, the number of positions to the right of any word during the pth round will be either same, or $n+3-p$ and $n+2-p$ respectively for $\rho_{1}, \rho_{2}$.

Proof. The starting configuration is $\left(i_{1}, j_{1}\right)$, the starting positions of the two words. Assume Spoiler chooses the word $\rho_{1}$, while Duplicator chooses $\rho_{2}$. Choosing the interval $I=(0,1)$, Spoiler invokes a $\mathrm{U}_{I}$ move and chooses one of the $a$ 's in $(0,1)$. In response, Duplicator chooses the only $a$ at 0.5 in $(0,1)$ in $\rho_{2}$. The possible configurations are those with time stamps $(0.5,0.5)$ or $(0.6,0.5)$. The configuration with time stamps $(0.6,0.5)$ is such that $i_{2}-j_{2}=3-2=1$, both words have exactly the same symbols in the future, at the same time points. Thus, Duplicator can achieve a configuration with identical time stamps, preserving the lag of one position. Let us now look at the configuration $\left(i_{2}, j_{2}\right)$ with time stamps $(0.5,0.5)$. Assume Spoiler continues to play in $\rho_{1}$, and chooses the $a$ at 0.6 by a $U_{(0,1)}$ move. In this case, 
Duplicator will choose the $a$ at 1.1, obtaining the configuration with time stamps $(0.6,1.1)$. The configuration $\left(i_{3}, j_{3}\right)$ with time stamps $(0.6,1.1)$ is such that $i_{3}=j_{3}$. Note that from $(0.6,1.1)$, Duplicator can always ensure an identical configuration $i_{p}=j_{p}, p \geq 3$ (Duplicator always moves the same number of positions as the Spoiler) or ensure a lag of one position (in this case, Spoiler moves ahead by more than one position and Duplicator also chooses the position with the same time stamp). Since the number of positions in $\rho_{1}$ is $n+3$ and that in $\rho_{1}$ is $n+2$, the number of positions to the right of any word during the $p$ th round will be either same, or $n+3-p$ and $n+2-p$ respectively.

If Spoiler starts playing from $\rho_{2}$, and chooses the $a$ at 0.5 using a $\mathrm{U}_{(0,1)}$ move, then Duplicator also chooses the $a$ at 0.5 in $\rho_{1}$. If Spoiler swaps the words at the end of this move, then Duplicator can achieve identical configurations for the rest of the game; otherwise, he can ensure a lag of atmost one position as seen above.

\section{$\mathrm{C}^{(0,1)} \mathrm{MTL} \subseteq \mathrm{C}^{0} \mathrm{MTL}$}

The containment of $\mathrm{C}^{(0,1)} \mathrm{MTL}$ in $\mathrm{C}^{0} \mathrm{MTL}$ follows from the fact that $\mathrm{C}^{(0,1)} \mathrm{MTL} \subseteq \mathrm{C}^{0} \mathrm{MTL}$. To show the strict containment, consider the formula $\varphi=\mathrm{C}_{(0,2)}^{\geq 2} a \in \mathrm{C}^{0} \mathrm{MTL}$. We show that for any choice of $n$ rounds and $k$ pebbles, we can find two words $\rho_{1}, \rho_{2}$ such that $\rho_{1} \models \varphi, \rho_{2} \not \models \varphi$, but $\rho_{1} \equiv{ }_{n, k}^{\mathrm{C}^{(0,1)} \mathrm{MTL}} \rho_{2}$. Consider the words $\rho_{1}=(a, 0)(a, 1.8)(a, 1.9) W$ and $\rho_{2}=(a, 0)(a, 1.9) W$ where $W$ is $(a, 2.1)(a, 2.1+\delta) \ldots,(a, 2.1+n k \delta)$ where $\delta<<\frac{1}{2 n k}$ such that $2.1+n k \delta<2.2$. Clearly, $\rho_{1} \models \varphi$ while $\rho_{2} \not \models \varphi$.

Proposition 3. In any round $p$ of the $\mathrm{C}^{(0,1)} \mathrm{MTL}$ game, Duplicator can always ensure an identical configuration $\left(i_{p}, j_{p}\right)$ or ensure that $0 \leq i_{p}-j_{p} \leq 1$. If $i_{p}-j_{p}=1$ and $j_{p} \geq 2$, then for all $q>p$, Duplicator can ensure that $0 \leq i_{q}-j_{q} \leq 1$. Further, the number of positions to the right of any word during the pth round will be either same, or $n k+4-p$ and $n k+3-p$ respectively.

Proof. The initial configuration is $\left(i_{1}, j_{1}\right)$ with time stamps $(0,0)$. Assume Spoiler picks $\rho_{2}$ while Duplicator chooses $\rho_{1}$. The first move cannot be a counting move, since there are no points in $(0,1)$ in both the words. Spoiler invokes an $U_{(1,2)}$ move and comes to 1.9 in $\rho_{2}$, while duplicator comes to 1.8 in $\rho_{1}$ (note that if Spoiler comes to a point $>1.9$, Duplicator comes to the point with the same time stamp). There are no inbetween points to be chosen in an until part, so the configuration is $\left(i_{2}, j_{2}\right)$ with time stamps $(1.8,1.9)$. This configuration is such that $i_{2}=j_{2}$, and the number of positions on the right are respectively $n k+2$ and $n k+1$. Lets consider the $2 \mathrm{cnd}$ round starting with $\left(i_{2}, j_{2}\right)$ having time stamps $(1.8,1.9)$. Assume Spoiler chose $\rho_{1}$ and a point $i_{2}^{\prime}$ with $t_{i_{2}^{\prime}}>1$.8. In response, Duplicator will choose a point $j_{2}^{\prime}$ with $t_{j_{2}^{\prime}}>1.9$. If $t_{i_{2}^{\prime}}$ is 1.9 , then $t_{j_{2}^{\prime}}$ is 2.1 with $i_{2}^{\prime}=j_{2}^{\prime}$. If $t_{i_{2}^{\prime}}>1.9$, then $t_{j_{2}^{\prime}}>2.1$ and Duplicator can ensure $t_{i_{2}^{\prime}}=t_{j_{2}^{\prime}}$. When Spoiler pebbles positions between $i_{2}$ and $i_{2}^{\prime}$, Duplicator can pebble in such a way that either $i_{3}=j_{3}$, or $t_{i_{3}}=t_{j_{3}}$, and $i_{3}-j_{3}=1$. When $t_{i_{3}}=t_{j_{3}}$, the number of points to the right is the same in both the words. In the case $t_{i_{2}^{\prime}}=1.9$, and $t_{j_{2}^{\prime}}=2.1$, we obtain $i_{3}=j_{3}$ with $n k+1$ and $n k$ positions respectively on the right in $\rho_{1}, \rho_{2}$. 
Assume that at the start of the $p$ th round, we have the configuration $\left(i_{p}, j_{p}\right)$ with $i_{p}=j_{p}$, and having respectively $n k+4-p$ and $n k+3-p$ positions to the right in $\rho_{1}, \rho_{2}$. Assume further that Spoiler chooses $i_{p}^{\prime}>i_{p}$, and Duplicator chooses $j_{p}^{\prime}>j_{p}$ as part of the $p$ th round's play. If $i_{p}^{\prime}>i_{p}+1$, then Duplicator chooses $j_{p}^{\prime}$ such that $t_{i_{p}^{\prime}}=t_{j_{p}^{\prime}}$, from where the number of positions on the right is the same in both words. Even if Spoiler decides to play a full until round by choosing a point $j_{p}^{\prime \prime}$ in between $j_{p}$ and $j_{p}^{\prime}$, Duplicator can always choose $i_{p}^{\prime \prime}$ having the same time stamp as $i_{p}^{\prime \prime}$. If $i_{p}^{\prime}=i_{p}+1$, then Duplicator has to choose $j_{p}^{\prime}=j_{p}+1$, and in this case, the lag of one position continues to the next configuration with $i_{p+1}=i_{p}^{\prime}$ and $j_{p+1}=j_{p}^{\prime}$. We then have respectively $n k+3-p$ and $n k+4-p$ positions to the right in $\rho_{1}, \rho_{2}$. In the case $i_{p}-j_{p}=1$, we have $t_{i_{p}}=t_{j_{p}}$. In this case, Duplicator can always ensure $i_{p+1}-j_{p+1}=1$, and the number of positions to the right are same in both the words.

\section{G Proof of Lemma 4}

Consider the formula $\varphi=\diamond_{(0,1) \#_{a} \geq 3} b \in \mathrm{TMTL}$. We show that for any choice of $n$ rounds and $k$ pebbles, we can find two words $\rho_{1}, \rho_{2}$ such that $\rho_{2} \models \varphi, \rho_{1} \not \models \varphi$, but $\rho_{1} \equiv{ }_{n, k}^{\mathrm{CMTL}} \rho_{2}$.

Let $l \in \mathbb{N}$ be the maximum constant used by Spoiler in the set $I_{\nu}$ of permissible intervals. Let $K=n l k+n l, \epsilon<\frac{1}{(10)^{10 n k}}$ and $\kappa=n k \epsilon$. Let $0.1>>n k \delta$ and $\delta>\kappa$. Design of Words

1. Consider the word $\rho_{1}$ of length $K+1$. Each unit interval $(i, i+1)$ in $\rho_{1}$, $0 \leq i \leq K$ is composed of 3 blocks $A_{i}, B_{i}, C_{i}$, one after the other.

- Block $A_{i}$ has the points $x_{i 1}=i+0.1+\epsilon+i \delta, y_{i 1}=i+0.1+\kappa+i \delta, z_{i 1}=i+0.2+i \delta$

- Block $B_{i}$ has the points $x_{i 2}=i+0.3+\epsilon+i \delta, y_{i 2}=i+0.3+\kappa+i \delta, z_{i 2}=i+0.4+i \delta$, and

- Block $C_{i}$ has the points $x_{i 3}=i+0.5+\epsilon+i \delta, y_{i 3}=i+0.5+\kappa+i \delta, z_{i 3}=0.9+i \delta$.

- Moreover, there are $p>>2 n l k$ points in between $x_{i j}$ and $y_{i j}$ for $1 \leq j \leq 3$. $\sigma_{z_{i j}}=a$ for all $i, j$, the points $x_{i j}, y_{i j}$ as well as all points between them are marked $b$.

- It can be seen that the blocks $A_{i}, B_{i}, C_{i}$ shift to the right by $\delta$, as $i$ increases from 0 to $K$.

2. The word $\rho_{2}$ has also length $K+1$. Each unit interval $(i, i+1)$ (except the last one) in $\rho_{2}$ is composed of 4 blocks $A_{i}^{\prime}, B_{i}^{\prime}, C_{i}^{\prime}, D_{i}^{\prime}$ one after the other.

- Block $A_{i}^{\prime}$ has the points $x_{i 1}^{\prime}=i+0.1+\epsilon+i \delta, y_{i 1}^{\prime}=i+0.1+\kappa+i \delta, z_{i 1}^{\prime}=i+0.2+i \delta$,

- Block $B_{i}^{\prime}$ has the points $x_{i 2}^{\prime}=i+0.3+\epsilon+i \delta, y_{i 2}^{\prime}=i+0.3+\kappa+i \delta, z_{i 2}^{\prime}=i+0.4+i \delta$

- Block $C_{i}^{\prime}$ has the points $x_{i 3}^{\prime}=i+0.5+\epsilon+i \delta$, $y_{i 3}^{\prime}=i+0.5+\kappa+i \delta, z_{i 3}^{\prime}=0.9+i \delta$,

- Block $D_{i}^{\prime}$ has the points $x_{i 4}^{\prime}=i+0.99+\epsilon+i \delta, y_{i 4}^{\prime}=i+0.99+\kappa+i \delta$. 
- Moreover, there are $p>>2 n l k$ points in between $x_{i j}$ and $y_{i j}$ for $1 \leq j \leq 4$. $\sigma_{z_{i j}^{\prime}}=a$ for all $i, j$, and the points $x_{i j}, y_{i j}$ as well as all points between them are marked $b$.

- It can be seen that the blocks $A_{i}^{\prime}, B_{i}^{\prime}, C_{i}^{\prime}$ and $D_{i}^{\prime}$ shift to the right by $\delta$, as $i$ increases from 0 to $K$.

- The last unit interval $(K-1, K)$ has only 3 blocks of $b$ 's starting respectively at $x_{K-11}^{\prime}, x_{K-12}^{\prime}$ and $x_{K-13}^{\prime}$ and ending at $y_{K-11}^{\prime}, y_{K-12}^{\prime}$ and $y_{K-13}^{\prime}$. The $3 a$ 's occur at $z_{K-1}, z_{K-12}$ and $z_{K-13}$.

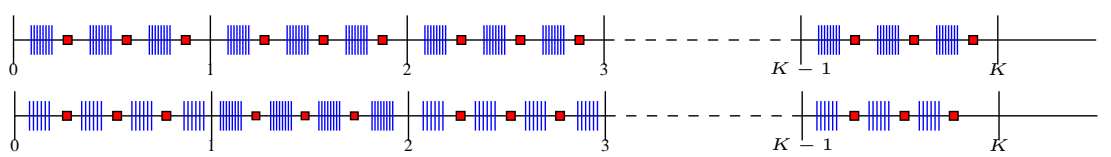

Fig. 5. The red square represents $a$, the bunch of blue lines represents a bunch of $b$ 's. There are $3 a$ 's in each unit interval of both $\rho_{1}$ and $\rho_{2}$. The difference is that $\rho_{1}$ has 3 blocks of $b$ 's in each unit interval, while $\rho_{2}$ has 4 blocks of $b$ 's in each unit interval except the last. Clearly, $\rho_{2} \models \varphi, \rho_{1} \not \models \varphi$. The time stamp of the three $a$ 's are $z_{i 1}, z_{i 2}$ and $z_{i 3}$ respectively in the $i$ th unit interval of $\rho_{1}$. Likewise, the $j$ th bunch of $b$ 's in the $i$ th unit interval begins with time stamp $x_{i j}$ and ends with time stamp $y_{i j}$. In $\rho_{2}$ we have $z_{i j}^{\prime}, x_{i j}^{\prime}$ and $y_{i j}^{\prime}$.

Clearly, $\rho_{1}, \rho_{2}$ have three occurrences of $a$ in each unit interval; however while $\rho_{1}$ has 3 blocks of $b$ 's in each unit interval with an $a$ after each $b$ block, $\rho_{2}$ has 4 blocks of $b$ 's in each unit interval, with the $3 a$ 's in between the $b$ blocks. Thus, $\rho_{2} \models \varphi$ while $\rho_{1} \not \models \varphi$.

Topological Similarity of $\rho_{1}, \rho_{2}$ : Note that for any $i$, the catenation of the blocks $D_{i}^{\prime}$ and $A_{i+1}^{\prime}$ is topologically similar to the block $A_{i+1}$ : (i) both have a sufficiently long sequence of $b$ 's followed by an $a ; D_{i}^{\prime} A_{i+1}^{\prime}$ has $2 p+2 b$ 's followed by an $a$, while $A_{i+1}$ has $p+2 b$ 's followed by an $a$. Since $p>>2 n l k$, and the number of rounds is $n$, a bunch of $2 p+2 b$ 's is as good as a bunch of $p+2$ 's. (ii) Map $A_{1}^{\prime}, B_{1}^{\prime}$ and $C_{1}^{\prime}$ respectively to $A_{1}, B_{1}$ and $C_{1}$; map $D_{i}^{\prime} A_{i+1}^{\prime}$ to $A_{i+1}, B_{i+1}^{\prime}$ to $B_{i+1}$ and $C_{i+1}^{\prime}$ to $C_{i+1}$ for $i \geq 1$.

Segmented View of $\rho_{1}, \rho_{2}$ : We will refer to the unit interval $(i, i+1)$ for $i \geq 0$ in either word as the $(i+1)$ th segment. Thus, both the words have $K$ segments numbered $1, \ldots, K$. For a position $i_{p} \in \operatorname{dom}\left(\rho_{1}\right) \cup \operatorname{dom}\left(\rho_{2}\right), \operatorname{seg}\left(i_{p}\right)$ represents the segment containing $t_{i_{p}}$. For instance, if $t_{i_{p}}=5.3$, then the position $i_{p}$ is contained in segment 6 , or $\operatorname{seg}\left(i_{p}\right)=6$.

Copy-cat strategy: Consider the $p$ th round of the game with initial configuration $\left(i_{p}, j_{p}\right)$. If Duplicator can ensure that $\operatorname{seg}\left(i_{p+1}\right)-\operatorname{seg}\left(i_{p}\right)=\operatorname{seg}\left(j_{p+1}\right)-\operatorname{seg}\left(j_{p}\right)$, then we say that Duplicator has adopted a copy-cat strategy in the $p$ th round.

We will now play a $(n, k)-C M T L$ game and show that Duplicator wins. It is easy to see that Duplicator can respond to any of the $U_{I}$ moves of Spoiler by the choice of the words. 
Proposition 4. For an $n$ round CMTL game over the words $\rho_{1}, \rho_{2}$, the Duplicator always has a winning strategy such that for any $1 \leq p \leq n$, if $\left(i_{p}, j_{p}\right)$ is the initial configuration of the pth round, then $\left|\operatorname{seg}\left(i_{p}\right)-\operatorname{seg}\left(j_{p}\right)\right| \leq 1$. Moreover, when $\left|\operatorname{seg}\left(i_{p}\right)-\operatorname{seg}\left(j_{p}\right)\right|=1$, then there are atleast $(n-p)(l+1)$ segments to the right on each word after $p$ rounds, for all $1 \leq p \leq n$.

Proof. Assume that Spoiler initiates a $\mathrm{C}_{\bar{I}}^{\geq k}$ move on $\rho_{2}$. Then Spoiler places $k$ pebbles on $k$ positions of $\rho_{2}$ in the interval $I$ and in response, Duplicator pebbles $k$ positions in the same interval $I$ of $\rho_{1}$.

1. If Spoiler does not keep any pebble on the last $b$ block in any of the unit intervals spanning $I$, then Duplicator puts his pebbles exactly at the same positions as Spoiler, and obtains an identical configuration.

2. Choice of Pebbling: Assume that we have an identical configuration $\left(i_{p}, j_{p}\right)$. Let us look at Spoiler's placement of pebbles on some unit interval say $(g, g+1)$. Assume that Spoiler keeps some (say $l$ ) pebbles on the last $b$ block (say $D_{g}^{\prime}$ ), and $l^{\prime}$ pebbles on the remaining 3 blocks $A_{g}^{\prime}, B_{g}^{\prime}$ and $C_{g}^{\prime}$ of the unit interval $(g, g+1)$. In response, Duplicator places $l^{\prime}$ of his pebbles at identical positions on $A_{g}, B_{g}$ and $C_{g}$, and places $l$ pebbles on $A_{g}$. Duplicator will place these $l$ pebbles in the first half of $A_{g}$. Note that since the number of positions in each block is $2 n k>>l$, this is possible. This way, Duplicator keeps his $k$ pebbles on the same unit intervals as Spoiler. If Spoiler picks a pebble in Duplicator's word from any $B$ or $C$ block, then Duplicator will pick the same pebble from Spoiler's word. If Spoiler picks a pebble from $A_{g}$, then there are two possibilities: (i) either this pebble corresponds to a pebble kept by Spoiler on $A_{g}^{\prime}$, or (ii) this is one of the $l^{\prime}$ pebbles kept by Duplicator on $A_{g}^{\prime}$ in response to Spoiler's $l^{\prime}$ pebbles on $D_{g}^{\prime}$. In case of (i), Duplicator simply picks the corresponding pebble from $A_{g}^{\prime}$, obtaining an identical configuration, while in case (ii), Duplicator picks the corresponding pebble from $D_{g}^{\prime}$. This gives a configuration $\left(i_{p+1}, j_{p+1}\right)$ with $i_{p+1}$ being a position in $A_{g}$, and $j_{p+1}$ in $D_{g}^{\prime}$. So far, there is no lag in the segments, $\operatorname{seg}\left(i_{p+1}\right)=g+1=\operatorname{seg}\left(j_{p+1}\right)$.

3. Consider any $\mathrm{U}_{I}$ move or $\mathrm{C}_{\bar{I}}^{\geq k}$ move that Spoiler launches on either of the words from $\left(i_{p+1}, j_{p+1}\right)$. Recall that $\operatorname{seg}\left(i_{p+1}\right)=g+1=\operatorname{seg}\left(j_{p+1}\right), i_{p+1} \in A_{g}$, $j_{p+1} \in D_{g}^{\prime}$.

(a) If Spoiler moves to some point in $A_{h}^{\prime}$ (in segment $h+1$ ), then Duplicator will move to some point in $A_{h-1}$ (in segment $h$ ). This is possible since for any interval $I, y_{h 1}^{\prime}-x_{g 4}^{\prime} \in I$ iff $y_{h-1}-x_{g 1} \in I$.

(b) If Spoiler moves to some point in $B_{h}^{\prime}$ (in segment $h+1$ ), then Duplicator will move to some point in $B_{h-1}$ (in segment $h$ ). This is possible since for any interval $I, y_{h 2}^{\prime}-x_{g 4}^{\prime} \in I$ iff $y_{h-12}-x_{g 1} \in I$.

(c) If Spoiler moves to some point in $C_{h}^{\prime}$ (in segment $h+1$ ), then Duplicator will move to some point in $C_{h-1}$ (in segment $h$ ). This is possible since for any interval $I, y_{h 3}^{\prime}-x_{g 4}^{\prime} \in I$ iff $y_{h-13}-x_{g 1} \in I$.

(d) If Spoiler moves to some point in $D_{h}^{\prime}$ (in segment $h+1$ ), then Duplicator will move to some point in $A_{h}$ (in segment $h+1$ ). This is possible since for any interval $I, y_{h 4}^{\prime}-x_{g 4}^{\prime} \in I$ iff $y_{h 1}-x_{g 1} \in I$. 
Cases (a)-(c) creates a lag of one segment between the two words, while (d) is similar to $\left(i_{p+1}, j_{p+1}\right)$. From (d), we can achieve any one of cases (a)-(d) listed above. Let us hence look at cases (a)-(c), to understand the potential future configurations.

In cases (a)-(c), when Spoiler pebbles $k$ positions between segments $g+1$ and $h+1$ in $\rho_{2}$, Duplicator pebbles $k$ positions between segments $g+1$ and $h$ in $\rho_{1}$. The choice of pebbling is as described in item 2 above:

(i) whenever Spoiler pebbles positions in block $D_{s}^{\prime}$ of segment $g+1 \leq s+1<h+1$ Duplicator pebbles positions in the first half of block $A_{s}$. If Spoiler picks one of these pebbles from $A_{s}$, then Duplicator chooses the corresponding pebble from $D_{s}^{\prime}$, thereby obtaining a configuration $\left(i_{q}, j_{q}\right)$ with $\operatorname{seg}\left(i_{q}\right)=\operatorname{seg}\left(j_{q}\right)=s+1$, with $i_{q} \in A_{s}, j_{q} \in D_{s}^{\prime}$. This configuration is exactly same as the one described in $3(\mathrm{~d})$ above.

(ii) whenever Spoiler places pebbles on $A_{s}^{\prime}, B_{s}^{\prime}$ and $C_{s}^{\prime}$, Duplicator places his pebbles on $A_{s}, B_{s}$ and $C_{s}$ respectively, for $s<h+1$. If Spoiler picks one of these pebbles from $A_{s}, B_{s}$ or $C_{s}$, then Duplicator chooses the corresponding pebble respectively from $A_{s}^{\prime}, B_{s}^{\prime}$ or $C_{s}^{\prime}$, obtaining a configuration $\left(i_{q}, j_{q}\right)$ with $i_{q} \in X_{s}$ iff $j_{q} \in X_{s}^{\prime}$ for $s<h+1$ and $X \in\{A, B, C\}$. $\operatorname{seg}\left(i_{q}\right)=\operatorname{seg}\left(j_{q}\right)=s+1$. In fact, this is an identical configuration.

(iii) whenever Spoiler keeps his pebbles on $A_{h+1}^{\prime}, B_{h+1}^{\prime}$ and $C_{h+1}^{\prime}$, Duplicator keeps his pebbles on $A_{h}, B_{h}$ and $C_{h}$ respectively. If Spoiler picks one of these pebbles from $A_{h}, B_{h}$ or $C_{h}$ then Duplicator chooses the corresponding pebble respectively from $A_{h+1}^{\prime}, B_{h+1}^{\prime}$ or $C_{h+1}^{\prime}$, obtaining a configuration $\left(i_{q}, j_{q}\right)$ with $\operatorname{seg}\left(i_{q}\right)=h+1, \operatorname{seg}\left(j_{q}\right)=h+2$ with $i_{q} \in X_{h}$ and $j_{q} \in X_{h+1}^{\prime}$ for $X \in\{A, B, C\}$. There is a lag of one segment here.

Cases (i) and (ii) have been explored before. Let us now explore case (iii), which gives the configuration $\left(i_{q}, j_{q}\right)$ with $i_{q} \in X_{h}, j_{q} \in X_{h+1}^{\prime}, X \in\{A, B, C\}$.

(a) Let $i_{q} \in A_{h}, j_{q} \in A_{h+1}^{\prime}$. Clearly, if Spoiler moves to $A_{d}^{\prime}, B_{d}^{\prime}$ or $C_{d}^{\prime}$, for $d \geq h+1$, Duplicator moves respectively to $A_{d-1}, B_{d-1}$ or $C_{d-1}$. Pebbling and picking a pebble here will give rise to a configuration as seen in (i), (ii) or (iii) above. The interesting case is when Spoiler moves from $j_{q}$ to a point in some $D_{d}^{\prime}$. Since there are no $D$ blocks in $\rho_{1}$, Duplicator moves to a point in $C_{d-1}$. Note that this is possible since $y_{d-13}-x_{h-11} \in I$ iff $y_{d 4}^{\prime}-x_{h 1}^{\prime} \in I$. Further, after pebbling, when Spoiler picks a pebble, Duplicator can either ensure an identical configuration, or a configuration as in 3(d).

(b) Let $i_{q} \in B_{h}, j_{q} \in B_{h+1}^{\prime}$. Clearly, if Spoiler moves to $A_{d}^{\prime}, B_{d}^{\prime}$ or $C_{d}^{\prime}$, Duplicator moves respectively to $A_{d-1}, B_{d-1}$ or $C_{d-1}$. Pebbling and picking a pebble here will give rise to a configuration as seen in (i), (ii) or (iii) above. The interesting case is when Spoiler moves from $j_{q}$ to a point in some $D_{d}^{\prime}$. Since there are no $D$ blocks in $\rho_{1}$, Duplicator moves to a point in $A_{d}$. Note that this is possible since $y_{d 1}-x_{h-12} \in I$ iff $y_{d 4}^{\prime}-x_{h 2}^{\prime} \in I$. Further, after pebbling, when Spoiler picks a pebble, Duplicator can either ensure an identical configuration, or a configuration as in 3(d). The case when $i_{q} \in C_{h}, j_{q} \in C_{h+1}^{\prime}$ is similar to the above : Duplicator can either preserve the lag or move to $A_{d}$ whenever Spoiler moves to $D_{d}^{\prime}$. This is possible since $y_{d 1}-x_{h-13} \in I$ iff $y_{d 4}^{\prime}-x_{h 3}^{\prime} \in I$. 
Thus, the possible configurations are (i) identical configurations $\left(i_{p}, j_{p}\right)$ with $i_{p} \in X_{h}$ iff $j_{p} \in X_{h}^{\prime}$ with $X \in\{A, B, C\}$, and no segment lag, or (ii) configurations with no segment lag of the form $\left(i_{p}, j_{p}\right)$ with $i_{p} \in A_{h}, j_{p} \in D_{h}^{\prime}$, or (iii) configurations with lag of one segment of the form $\left(i_{p}, j_{p}\right)$ with $i_{p} \in X_{h}, j_{p} \in X_{h+1}^{\prime}$ with $X \in\{A, B, C\}$. If Spoiler always chooses bounded intervals (of length $\leq l$ ), then Duplicator respects his segment lag of 1 , and the maximum number of segments that can be explored in either word is atmost $n l<K$. In this case, there are atleast $K-p l \geq n l k+n l-p l \geq$ $(n-p)(l+1)$ segments to the right of $\rho_{1}$ and $K-p l+1$ segments to the right of $\rho_{2}$ after $p$ rounds.

If Spoiler chooses an unbounded interval of length $>l$ in any round, then Duplicator moves ahead only by $l+1$ segments. The pebbles Spoiler drops in his $D^{\prime}$ blocks can be accommodated by Duplicator in the $A$ blocks of these $l+1$ segments since, the number of points in the $A$ blocks are much more than $2 n k l$, and atmost $k$ pebbles are placed in a round. Having done this, Duplicator can either enforce an identical configuration, or obtain the configuration $\left(i_{p}, j_{p}\right)$ with $i_{p} \in A_{h}$ and $j_{p} \in D_{h}^{\prime}$. Since we have seen that Duplicator can always replicate Spoiler's move from configurations of this kind $\left(i_{p}, j_{p}\right) i_{p} \in A_{h}$ and $j_{p} \in D_{h}^{\prime}$, for the remaining $n-p$ rounds either we have $\operatorname{seg}\left(i_{q}\right)=\operatorname{seg}\left(j_{q}\right), q>p$, or $\left|\operatorname{seg}\left(i_{q}\right)-\operatorname{seg}\left(j_{q}\right)\right|=1$ for all $q>p$. Thus, whenever an unbounded interval is used, the segments match, and Duplicator ensures that the maximum segments covered after any $p$ rounds is $\leq p(l+1)$. This ensures that after $n$ rounds, we cover atmost $n(l+1)$ segments on either word. Thus, Duplicator can always replicate moves of the Spoiler and there are $K-p(l+1) \geq n l k+n l-p l-p$ $\geq n-p+(n l-p l)=(n-p)(l+1)$ segments to the right of each word after $p$ rounds for all $p \leq n$.

\section{H Oversampling : Relevant Lemmas}

If $\psi$ is over $\Sigma \cup X$, then the relativization of $\psi$ with respect to $\Sigma$ is denoted $O N F_{\Sigma}(\psi)$ [3] and defined inductively as follows: If $\psi=a \in \Sigma$, then $O N F_{\Sigma}(\psi)=(a \wedge \bigvee \Sigma)$. Likewise, if $\psi=\varphi_{1} \mathrm{U} \varphi_{2}$, then $O N F_{\Sigma}(\psi)=\left[\left(a c t \rightarrow O N F_{\Sigma}\left(\varphi_{1}\right)\right)\right.$ $\mathrm{U}\left(\right.$ act $\left.\left.\wedge O N F_{\Sigma}\left(\varphi_{2}\right)\right)\right]$ where $a c t=\bigvee \Sigma$. It can then be seen [6] that for $\zeta_{1}=O N F_{\Sigma_{1}}\left(\psi_{1}\right)$ and $\zeta_{2}=O N F_{\Sigma_{2}}\left(\psi_{2}\right)$, with $\Sigma_{1}=\Sigma \cup X_{1}, \Sigma_{2}=\Sigma \cup X_{2}$ and disjoint $X_{1}, X_{2}$, if $\varphi_{1}=\exists \downarrow X_{1} \cdot \zeta_{1}$ and $\varphi_{2}=\exists \downarrow X_{2} \cdot \zeta_{2}$, then $\varphi_{1} \wedge \varphi_{2}=\exists \downarrow\left(X_{1} \cup X_{2}\right) .\left(\zeta_{1} \wedge \zeta_{2}\right)$. The following lemmas are from [6].

Lemma 12. Consider formulae $\varphi_{1}, \varphi_{2}$ built from $\Sigma$. Let $\psi_{1}, \psi_{2}$ be formulae built from $\Sigma \cup X_{1}$ and $\Sigma \cup X_{2}$ respectively. Let $X=X_{1} \cup X_{2}, \Sigma_{i}=\Sigma \cup X_{i}$ for $i=1,2$, and $X_{1} \cap X_{2}=\emptyset$. Let $\zeta_{1}=O N F_{\Sigma_{1}}\left(\psi_{1}\right)$ and $\zeta_{2}=O N F_{\Sigma_{2}}\left(\psi_{2}\right)$. Then, $\varphi_{1}=\exists \downarrow X_{1} . \zeta_{1}$ and $\varphi_{2}=\exists \downarrow X_{2} \cdot \zeta_{2}$ implies $\varphi_{1} \wedge \varphi_{2}=\exists \downarrow X .\left(\zeta_{1} \wedge \zeta_{2}\right)$.

Lemma 13. Let $\varphi \in \mathrm{CMTL}$ be built from $\Sigma$, and $W$ be the set of witness variables obtained while flattening $\varphi$. Then $\varphi=\exists \downarrow W . O N F_{\Sigma}\left(\varphi_{\text {flat }}\right)$.

\section{Proof of Lemma 5}

We want to express that the number of times $b$ is true in the region $[l, \infty)$ of any timed word $\rho$ is $\geq n$. 
Let $\left\{\varphi_{1}, \varphi_{2}, \zeta, n-1\right\}$ denote the formula $\varphi_{1} \mathrm{U}\left[\varphi_{2} \wedge\left(\varphi_{1} \mathrm{U}\left[\varphi_{2} \wedge \ldots\left(\varphi_{1} \mathrm{U} \zeta\right) \ldots\right]\right)\right]$, where the depth of the nested until is $n-1$. The formula $\psi=\diamond_{[l, \infty)}[b \wedge\{\neg b, b, b, n-1\}]$ in MTL captures this requirement. Clearly, $\psi$ evaluates to true on timed words where there is a point in $[l, \infty)$ where $b$ is true, and continues to be true for atleast $n$ times.

\section{J Proof of Lemma 6(2)}

We construct the $(\Sigma \cup W, X)$-simple extension $\rho^{\prime}$ from $\rho$ exactly as we did in Lemma 6(1), using the same set of new propositions $X=\left\{b_{0}, \ldots, b_{n}\right\}$. We use these propositions as counters in the same way as in Lemma 6 . Note that if the number of occurrences of $b$ in some segment of the timed word $\rho^{\prime}$ is less than $n$ then at least one of the counters $b_{i}$ will be missing. A point $h$ should be marked with witness $a$ iff there exist a point $j>h$ with $y \in \sigma_{j}, t_{j}-t_{h} \in I, x \in \sigma_{i}$ for $h<i<j$, and the number of times $b$ has occurred in $\left[t_{h}, t_{j}\right]$ is less than $n$. Checking the number of occurrences of $b$ to be $<n$ amounts to checking that at least one of the propositions from $X$ is missing from $\left[t_{h}, t_{j}\right]$. The formula $\lambda=\square^{\mathrm{w}}\left[a \leftrightarrow\left[\bigvee_{k=1}^{n}\left(x \wedge \neg b_{k}\right)\right] \mathrm{U}_{I} y\right]$ captures all positions where this is true; all such psoitions are marked $a$. Thus the formula $\zeta=\delta \wedge \lambda$ is the required formula in MTL.

\section{K Timed Propositional Temporal Logic (TPTL)}

Syntax of TPTL: $\varphi::=a(\in \Sigma) \mid$ true $|\varphi \wedge \varphi| \neg \varphi|\varphi \mathrm{U} \varphi| \mathrm{O} \varphi|y . \varphi| y \in I$ where $y$ is a clock variable. There is a finite set $\mathcal{C}$ of clock variables progressing at the same rate, and $I$ is an interval of the form $<a, b>a, b \in \mathbb{N}$ with $<\in\{(,[\}$ and $>\in\{])$,$\} . TPTL$ is interpreted over words in $T \Sigma^{*}$. The truth of a formula is interpreted at a position $i \in \mathbb{N}$ along the word. For a timed word $\rho=\left(\sigma_{1}, t_{1}\right) \ldots\left(\sigma_{n}, t_{n}\right)$, we define the satisfiability relation, $\rho, i, \nu \models \phi$ saying that the formula $\phi$ is true at position $i$ of the timed word $\rho$ with a valuation $\nu$ of all the clock variables at $i . \nu(x)$ is the valuation of clock $x$. The notation $\nu\left[x \leftarrow t_{i}\right]$ represents replacing the valuation of $x$ with $t_{i}$.

$\rho, i, \nu \models a \leftrightarrow a \in \sigma_{i}$ and $\rho, i, \nu \models \neg \varphi \leftrightarrow \rho, i, \nu \not \models \varphi$

$\rho, i, \nu \models \varphi_{1} \wedge \varphi_{2} \leftrightarrow \rho, i, \nu \models \varphi_{1}$ and $\rho, i, \nu \models \varphi_{2}$

$\rho, i, \nu \models x . \varphi \leftrightarrow \rho, i, \nu\left[x \leftarrow t_{i}\right] \models \varphi$ and $\rho, i, \nu \models x \in I \leftrightarrow t_{i}-\nu(x) \in I$

$\rho, i, \nu \models \mathrm{O} \varphi \leftrightarrow \rho, i+1, \nu \models \varphi$

$\rho, i, \nu \models \varphi_{1} \mathrm{U} \varphi_{2} \leftrightarrow \exists j>i, \rho, j, \nu \models \varphi_{2}$, and $\rho, k, \nu \models \varphi_{1} \forall i<k<j$

$\rho$ satisfies $\phi$ denoted $\rho \models \phi$ iff $\rho, 1, \overline{0} \models \phi$. Here $\overline{0}$ is the valuation obtained by setting all clock variables to 0 . TPTL ${ }^{\mathrm{n}}$ denotes the class of TPTL formulae using $\leq n$ clocks. For example, $x$. $[a \wedge \diamond(b \wedge x \in(0,1) \wedge \diamond(b \wedge x \in(0,1)))]$ is a formula in $\mathrm{TPTL}^{1}$ which specifies that there are two $b$ 's within distance $(0,1)$ from $a$.

\section{K.1 CTMTL $\subset$ TPTL $^{1}$}

$\mathrm{CTMTL} \subseteq \mathrm{TPTL}^{1}$ : We show that we can encode both the $\mathrm{C}$ modality as well as UT modality in $\mathrm{TPTL}^{1}$. Consider the $\mathrm{C}$ modality $\mathrm{C}_{\bar{I}}^{\geq n} \varphi$. Recall that this formula holds 
good at a point $i$ in a timed word iff $\varphi$ evaluates to true $\geq n$ times in the interval $t_{i}+I$. We capture this in $\mathrm{TPTL}^{1}$ as follows:

- Let $2 \varphi_{1}, \varphi_{2}, \zeta, n-1 \int$ denote the formula $\varphi_{1} \mathrm{U}\left[\varphi_{2} \wedge\left(\varphi_{1} \mathrm{U}\left[\varphi_{2} \wedge \ldots\left(\varphi_{1} \mathrm{U} \zeta\right) \ldots\right]\right)\right]$, where the depth of the nested until is $n-1$. The TPTL formula $x . \diamond\left(x \in I \wedge\left(\varphi \wedge 2 \neg \varphi, \varphi, \varphi \wedge x \in I, n-1 \int\right)\right)$

evaluates to true at a position $i$ in any timed word $\rho$ iff there is a position $j>i$ such that $t_{j} \in t_{i}+I, \rho, j, \nu \models \varphi$ with $\nu \in I$, and there exists a point $k>j$ such that $\rho, k, \nu \models \varphi$ with $\nu \in I$, and there exist $n-2$ points $j<i_{1}<i_{2}<\cdots<i_{n-2}<k$ where $\varphi$ evaluates to true, and $\neg \varphi$ is true in $\left(j, i_{1}\right),\left(i_{1}, i_{2}\right), \ldots,\left(i_{n-2}, k\right)$. It is clear that the clock valuation at all these $n-2$ inbetween points satisfy $\nu \in I$ (since $\nu \in I$ at both $j, k$ ). Thus, we have obtained $n$ points in $t_{i}+I$ where $\varphi$ is true. Clearly, this captures the semantics of $\mathrm{C}_{\bar{I}}^{\geq n} \varphi$.

- The modality $\mathrm{C}_{I}^{<n} \varphi$ is obtained by negating $\mathrm{C}_{I}^{\geq n} \varphi$, while $\mathrm{C}_{I}^{=n} \varphi$ is written as a conjunction of $\mathrm{C}_{\bar{I}}^{\geq n} \varphi$ and $\mathrm{C}_{\bar{I}}^{\leq n} \varphi$.

Now we embed the UT modality $\varphi_{1} \mathrm{U}_{I, \eta} \varphi_{2}$ in $\mathrm{TPTL}^{1}$. Using Lemma11, we just have to show that the counting modality $\varphi_{1} \mathrm{U}_{I, \eta} \varphi_{2}$ where $\eta$ is free of conjunctions can be expressed in TPTL ${ }^{1}$. Let $\eta=\# \psi_{\psi} \geq c$. Then the formula

$x .\left(2 \varphi_{1} \wedge \neg \psi, \varphi_{1} \wedge \psi, \varphi_{1} \wedge \psi \wedge\left\{\varphi_{1} \mathrm{U}\left(\varphi_{2} \wedge x \in I\right)\right\}, c \int\right)$ is the formula in $\mathrm{TPTL}^{1}$ that captures $\varphi_{1} \mathrm{U}_{I, \eta} \varphi_{2}$ : clearly, this formula evaluates to true at a point $i$ iff there is a position $j>i$ such that at $j, \varphi_{2} \wedge x \in I$ evaluates to true (note that $x$ was reset at $i$ ), and all the way between $i$ and $j, \varphi_{1}$ evaluates to true. Further, we have a nested until of depth $c$, which witnesses $n$ points $i<i_{1}<\cdots<i_{n}<j$, such that $\varphi_{1} \wedge \psi$ evaluates to true at each $i_{j}$, and $\neg \psi$ evaluates to true in $\left(i_{j-1}, i_{j}\right)$. This process can be repeated to handle threshold formulae of counting depth $i>1$, by recursively replacing the threshold formulae at each level of $\eta$ by an appropriate $\mathrm{TPTL}^{1}$ formula. Finally, the untimed threshold counting modality introduced in Lemma 11 can be replaced in $\mathrm{TPTL}^{1}$ by a technique similar to that in [5].

To show the strict containment of CTMTL in $\mathrm{TPTL}^{1}$, we consider the formula $\varphi=x . \diamond[a \wedge x \in(0,1) \wedge \square[x \in(0,1) \rightarrow \neg b]] \in \mathrm{TPTL}^{1}$. The $\mathrm{TPTL}^{1}$ formula says that there is an $a$ in $(0,1)$, and the last symbol in $(0,1)$ is not a $b$. This formula was shown to be not expressible in MTL [8]. We show here that $\varphi$ cannot be expressed even with counting, that is in CTMTL. We show that for any choice of $n$ rounds and $k$ pebbles, we can find two words $\rho_{1}, \rho_{2}$ such that $\rho_{2} \models \varphi, \rho_{1} \not \models \varphi$, but $\rho_{1} \equiv_{n, k}^{\text {CTMTL }} \rho_{2}$. Let $p \in \mathbb{N}$ be such that $p n k>>k$ and $0<\delta<<\frac{1}{2 p n k}$.

1. Consider the word $\rho_{1}=\left((a b)^{p n k}(a b)^{p n k}, \tau\right)$ where the time stamps are as follows: the first $2 p n k$ symbols lie in the interval $(0,1)$, with the first time stamp $t_{1}=0.9$, $t_{2 p n k}=0.9+(2 p n k-1) \delta, t_{i+1}-t_{i}=\delta$ for all $0<i<2 p n k$. The remaining $2 p n k$ time stamps are such that $t_{2 p n k+1}=1.1, t_{4 p n k}=1.1+(2 p n k-1) \delta$ and $t_{i+1}-t_{i}=\delta$ for all $2 p n k+1<i<4 p n k$. By the choice of $\delta$, we have $1.1+(2 p n k-1) \delta<1.2$, and $0.9+(2 p n k-1) \delta<1$.

2. The second word is $\rho_{2}=\left((a b)^{p n k-1} a(b a)^{p n k} b, \tau^{\prime}\right)$, with time stamps $t_{i}^{\prime}=t_{i}+\delta$ for $1 \leq i \leq 2 p n k-1$ and $t_{2 p n k}^{\prime}=1.1-\delta>1, t_{i}^{\prime}=t_{i}$ for $2 p n k+1 \leq i \leq 4 p n k$.

3. In the case of $\rho_{1}$, the last time stamp $<1$ is $t_{2 p n k}=0.9+(2 p n k-1) \delta$ and the letter at that position is $\sigma_{2 p n k}=b$. For $\rho_{2}$, 
$t_{2 p n k-1}^{\prime}=t_{2 p n k-1}+\delta=t_{2 p n k}=0.9+(2 p n k-1) \delta$ is the last time stamp $<1$, and the letter at this position is $\sigma_{2 p n k-1}^{\prime}=a$. Hence, $\rho_{1} \not \models \varphi, \rho_{2} \models \varphi$.

4. While the last $b$ in $(0,1)$ of $\rho_{1}$ is at position $2 p n k$ with time stamp

$0.9+(2 p n k-1) \delta$, the last $b$ in $(0,1)$ of $\rho_{2}$ is at position $2 p n k-2$ with time stamp

$0.9+(2 p n k-2) \delta$.

We now show that in a $n, k$-CTMTL game over $\rho_{1}, \rho_{2}$, Duplicator wins. The main intuition here is that apart from the fact that there is a lag of one symbol across intervals $(0,1)$ and $(1,2)$, there is no difference between $\rho_{1}$ and $\rho_{2}$.

From the initial configuration $\left(i_{0}, j_{0}\right)$ with time stamps $(0,0)$, Spoiler initiates an $\mathbf{U}_{I}$ move or a $\mathrm{C}$ move or a UT move.

1. If Spoiler initiates a $\mathbf{U}_{(1,2)}$ move on $\rho_{1}$ and comes to the position $2 p n k+1$ with time stamp 1.1 as part of the $\nabla_{(1,2)}$ move, then Duplicator will come on $\rho_{2}$ to the position $2 p n k+1$ with the same time stamp $\sigma_{2 p n k+1}=a=\sigma_{2 p n k+1}^{\prime}$. So we have $i_{0}^{\prime}=2 p n k+1, j_{0}^{\prime}=2 p n k+1$. The future is identical in both words from this point. The interesting case is when Spoiler chooses to do the full until move or a $\mathrm{C}$ move or a UT move at $\left(i_{0}^{\prime}, j_{0}^{\prime}\right)$.

Consider the until move first. In this case, Spoiler chooses some position $1<h \leq 2 p n k$ in $\rho_{2}$. In this case, Duplicator will choose the same position in $\rho_{1}$. Even though the time stamps differ, all the points to the right in both $\rho_{1}, \rho_{2}$ lie in $(0,1)$ from $t_{h}$. Moreover, the number of points to the right are the same, with the same symbols. Hence, Duplicator wins. Now let us consider a $\mathrm{C}$ move from $\left(i_{0}^{\prime}, j_{0}^{\prime}\right)$. The only relevant move is $\mathrm{C}_{(0,1)}$, since all points to the right of $\left(i_{0}^{\prime}, j_{0}^{\prime}\right)$ lie in interval $(0,1)$. The number of points to the right of $\left(i_{0}^{\prime}, j_{0}^{\prime}\right)$ in both words are much larger than $k$. The number of points between $i_{0}$ and $i_{0}^{\prime}$ as well as $j_{0}$ and $j_{0}^{\prime}$ are both much $>>k$; infact the number of $a$ 's as well as $b$ 's are much more than $k$. Duplicator can place his pebbles at the same positions as Spoiler, and obtain an identical configuration. The argument is exactly same for a UT move from $\left(i_{0}^{\prime}, j_{0}^{\prime}\right)$. Again, the only relevant move is $\mathrm{U}_{(0,1), \eta}$.

2. Let us now look at the more interesting case when Spoiler initiates a $U_{(0,1)}$ move or a $\mathrm{C}_{(0,1)}$ on $\rho_{1}$ from $\left(i_{0}, j_{0}\right)$ and chooses the last symbol in $(0,1)$, the $b$ at position $i_{0}^{\prime}=2 p n k$ of $\rho_{1}$ with time stamp $0.9+(2 p n k-1) \delta$. In this case, Duplicator will choose the last $b$ in $(0,1)$ at position $j_{0}^{\prime}=2 p n k-2$ of $\rho_{2}$ with time stamp $0.9+(2 p n k-2) \delta$. In the case of $\mathbf{U}_{(0,1)}$ move, Spoiler can decide to end this move, in which case, the configuration will be $\left(i_{1}, j_{1}\right)$ with time stamps $(0.9+(2 p n k-1) \delta, 0.9+(2 p n k-2) \delta)$. If Spoiler decides to go ahead with the $\mathrm{U}$ move, and chooses a position $1<h<2 p n k-2$ in Duplicator's word, then Duplicator will pick the same position $1<h<2 p n k$ in Spoiler's word. This gives the identical configuration $\left(i_{1}, j_{1}\right)=(h, h)$. All the points to the right of $h$ in $\rho_{1}$, as well as all the points to the right of $h$ in $\rho_{2}$ lie in the interval $(0,1)$, since the time stamps are $t_{h}=0.9+(h-1) \delta$ and $t_{h}^{\prime}=0.9+h \delta$. Clearly, any move of Spoiler can be mimicked by Duplicator obtaining an identical configuration. In case of the $\mathrm{C}_{(0,1)}$ move between $i_{0}$ and $i_{0}^{\prime}$ and $j_{0}$ and $j_{0}^{\prime}$, it can be seen that since the number of $a$ 's and $b$ 's are $>>k$, Duplicator can place his pebbles at the same positions as Spoiler and obtain an identical configuration. 
3. Now consider the case of a UT move. Assume Spoiler initiates a $\mathrm{U}_{I, \eta}$ move with $I=(0,1)$ from $(0,0)$, and plays on $\rho_{1}$. As part of the $\diamond_{(0,1)}$ move, If Spoiler comes on the last position in $(0,1)$ which is the $b$, Duplicator will come on to the last $b$ in $(0,1)$. If the Spoiler continues with the counting move, then Spoiler keeps $k$ pebbles in the positions between 0 and $2 p n k$, while Duplicator keeps his $k$ pebbles between 0 and $2 p n k-2$ at identical positions in his own word. It can be seen as in the case of the $\mathrm{C}$ move that Duplicator can ensure an identical configuration.

4. The argument when Spoiler plays on $\rho_{2}$ is exactly the same. 